\title{
APPLICABILITY AND APPLICATIONS OF THE METHOD OF FUNDAMENTAL SOLUTIONS
}

\author{
YIORGOS-SOKRATIS SMYRLIS
}

\begin{abstract}
In the present work, we investigate the applicability of the method of fundamental solutions for the solution of boundary value problems of elliptic partial differential equations and elliptic systems. More specifically, we study whether linear combinations of fundamental solutions can approximate the solutions of the boundary value problems under consideration. In our study, the singularities of the fundamental solutions lie on a prescribed pseudo-boundary - the boundary of a domain which embraces the domain of the problem under consideration. We extend previous density results of Kupradze and Aleksidze, and of Bogomolny, to more general domains and partial differential operators, and with respect to more appropriate norms. Our domains may possess holes and their boundaries are only required to satisfy a rather weak boundary requirement, namely the segment condition. Our density results are with respect to the norms of the spaces $C^{\ell}(\bar{\Omega})$. Analogous density results are obtainable with respect to Hölder norms. We have studied approximation by fundamental solutions of the Laplacian, biharmonic and $m$-harmonic and modified Helmholtz and poly-Helmholtz operators. In the case of elliptic systems, we obtain analogous density results for the Cauchy-Navier operator as well as for an operator which arises in the linear theory of thermo-elasticity. We also study alternative formulations of the method of fundamental solutions in cases when linear combinations of fundamental solutions of the equations under consideration are not dense in the solution space. Finally, we show that linear combinations of fundamental solutions of operators of order $m \geq 4$, with singularities lying on a prescribed pseudo-boundary, are not in general dense in the corresponding solution space.
\end{abstract}

\section{INTRODUCTION}

Let $\mathcal{L}=\sum_{|\alpha| \leq m} a_{\alpha}(\boldsymbol{x}) D^{\alpha}$ be an elliptic partial differential operator in $\Omega \subset \mathbb{R}^{n}$ of order $m$. In Trefftz methods, the solution of the boundary value problem

$$
\begin{aligned}
& \mathcal{L} u=0 \quad \text { in } \Omega, \\
& \mathcal{B} u=\boldsymbol{f} \quad \text { on } \partial \Omega,
\end{aligned}
$$

where $\Omega$ is an open domain in $\mathbb{R}^{n}$ and $\mathcal{B} u=f$ is the boundary condition 1 , is approximated by linear combinations of particular solutions of (1.1a), provided that

Received by the editor December 18, 2007.

2000 Mathematics Subject Classification. Primary 35E05, 41A30, 65N35; Secondary 35G15, $35 \mathrm{~J} 40,65 \mathrm{~N} 38$.

Key words and phrases. Trefftz methods, method of fundamental solutions, fundamental solutions, elliptic boundary value problems, approximation by special function.

This work was supported by University of Cyprus grant \#8037-3/312-21005.

${ }^{1}$ Throughout this work, all vectors will be denoted in bold print.

(C) 2009 American Mathematical Society Reverts to public domain 28 years from publication 
such linear combinations are dense in the set of all solutions of this equation. Erich Trefftz presented this approach in 1926 [53] as a counterpart of Ritz's method. A typical Trefftz method is the approximation of harmonic (resp. holomorphic) functions by harmonic (resp. harmonic) polynomials. In his celebrated work 40], Mergelyan showed in 1952 that holomorphic functions in bounded simply connected domains in $\mathbb{C}$ can be approximated, in the sense of the uniform norm, by polynomials, whereas in the case of multi-connected domains they can be approximated by rational functions. Mergelyan's work is a culmination of a long series of works by Runge, J. L. Walsh, Lavrent'ev and Keldysh on approximations by polynomials and rational functions. As early as 1885, Runge 43 proved that holomorphic functions in an open domain $U$ of the complex plane can be approximated, uniformly in compact subsets of $U$, by rational functions.

In the method of fundamental solutions (MFS), the particular solutions of the partial differential equation under consideration are the fundamental solutions $\varphi(\boldsymbol{x}, \boldsymbol{y})$ of the corresponding partial differential operator. They satisfy

$$
\mathcal{L}_{\boldsymbol{x}} \varphi(\cdot, \boldsymbol{y})=\delta_{\boldsymbol{y}},
$$

for every $\boldsymbol{y}$, where the notation $\mathcal{L}_{x} \varphi$ signifies that $\varphi$ is differentiated with respect to $\boldsymbol{x}$ and $\delta_{\boldsymbol{y}}$ is the Dirac measure with unit mass at $\boldsymbol{y}$, in the sense of distributions, i.e.,

$$
\int_{\mathbb{R}^{n}} \varphi(\boldsymbol{x}, \boldsymbol{y}) \mathcal{L}^{\star} \psi(\boldsymbol{x}) d \boldsymbol{x}=\psi(\boldsymbol{y}),
$$

for every $\psi \in C_{0}^{\infty}\left(\mathbb{R}^{n}\right)$, where $\mathcal{L}^{\star} u=\sum_{|\alpha| \leq m}(-1)^{|\alpha|} D^{\alpha}\left(a_{\alpha} u\right)$. The operator $\mathcal{L}^{\star}$ is the adjoint of $\mathcal{L}$. In particular, if $\mathcal{L}$ is elliptic with constant coefficients, then $\varphi(\cdot, \boldsymbol{y})$ is real analytic in $\mathbb{R}^{n} \backslash\{\boldsymbol{y}\}$ and satisfies, in the classical sense, $\mathcal{L}_{\boldsymbol{x}} \varphi(\boldsymbol{x}, \boldsymbol{y})=0$ for every $\boldsymbol{x} \in \mathbb{R}^{n} \backslash\{\boldsymbol{y}\}$. (See Rudin [42].) The point $\boldsymbol{y}$ is known as the singularity of $\varphi$. In the MFS, the singularities of the fundamental solutions lie outside the domain $\Omega$. The fundamental solutions were first introduced by Laurent Schwartz 44. Malgrange [38] and Ehrenpreis [15] independently established in 1955-56 the existence of fundamental solutions for partial differential operators with constant coefficients. (A simple proof can be found in [42, Theorem 8.15].) Also, if $\mathcal{L}$ has partial differential operators with constant coefficients, then $\mathcal{L}$ possesses a fundamental solution of the form $\varphi(\boldsymbol{x}, \boldsymbol{y})=e(\boldsymbol{x}-\boldsymbol{y})$. The fundamental solutions of elliptic operators have the following property ([57]):

Proposition 1. If $\mathcal{L}$ is an elliptic operator with constant coefficients of order $m$ in $\mathbb{R}^{n}$ and $e=e(\boldsymbol{x})$ is a fundamental solution of $\mathcal{L}$, then $D^{\alpha} e \in L_{\text {loc }}^{1}\left(\mathbb{R}^{n}\right)$, for every $|\alpha|<m$.

Felix Browder showed in 1962 that linear combinations of fundamental solutions of elliptic operators with singularities in an arbitrary open set $U$ outside a connected domain $\bar{\Omega}$ are dense, in the sense of the uniform norm, in the space

$$
\mathcal{X}=\left\{u \in C^{m}(\Omega): \mathcal{L} u=0 \text { in } \Omega\right\} \cap C(\bar{\Omega}) .
$$

Browder's proof relies on a duality argument. (See [10, Theorem 3].) Browder's result extends to any partial differential operator $\mathcal{L}$, with the property that its adjoint $\mathcal{L}^{\star}$ satisfies the Condition of uniqueness for the Cauchy problem in the small in $\Omega$, namely: 
$(\mathrm{U})_{s}$. If $u \in C^{m}(V)$, where $V$ is an open connected subset of $\Omega$ with $\mathcal{L}^{\star} u=0$ and if $u$ vanishes in a nonempty open subset of $V$, then $u$ vanishes everywhere in $V$.

Elliptic operators with real analytic coefficients satisfy condition $(\mathrm{U})_{s}$ since the solutions of the corresponding homogeneous equations are real analytic functions. (See [24, Corollary 4.4.4].) A detailed survey on the extensions of Browder's work and approximations of solutions of elliptic equations by solutions of the same equations in larger domains can be found in 52 .

Weinstock [57] extended Browder's density results to bounded domains which satisfy a weaker regularity requirement, the segment condition. In particular, he showed that, if $\Omega$ is such a domain and $\mathcal{L}$ is an elliptic operator with constant coefficients of order $m$, then for any $k<m$, the solutions of $\mathcal{L} u=0$ in $\Omega$, which belong to $C^{k}(\bar{\Omega})$, can be approximated, in the norm of $C^{k}(\bar{\Omega})$, by functions which are solutions of $\mathcal{L} u=0$ in a neighborhood of $\bar{\Omega}$.

The MFS was introduced by Kupradze and Aleksidze [33] in 1963 as the method of generalized Fourier series (метод обобщенных рядов Фурье). In a typical application to the Dirichlet problem for Laplace's equation,

$$
\left\{\begin{aligned}
\Delta u=0 & \text { in } \Omega \\
u=f & \text { on } \partial \Omega
\end{aligned}\right.
$$

the function $\varphi(\boldsymbol{x}, \boldsymbol{y})=e_{1}(\boldsymbol{x}-\boldsymbol{y})$, where

$$
e_{1}(\boldsymbol{x})= \begin{cases}-\frac{\log |\boldsymbol{x}|}{2 \pi}, & \text { if } n=2, \\ -\frac{|\boldsymbol{x}|^{2-n}}{(2-n) \omega_{n-1}}, & \text { if } n>2\end{cases}
$$

is a fundamental solution of the Laplacian (more precisely of $-\Delta$ which is an elliptic operator according to the definition), where $\omega_{n-1}$ is the area of the surface of the unit sphere $S^{n-1}$ in $\mathbb{R}^{n}$ and $|\cdot|$ is the Euclidean norm in $\mathbb{R}^{n}$. In the MFS, the solution of (1.5) is approximated by a finite linear combination of the form

$$
u_{N}(\boldsymbol{x} ; \boldsymbol{c})=\sum_{j=1}^{N} c_{j} \varphi\left(\boldsymbol{x}, \boldsymbol{y}_{j}\right)=\sum_{j=1}^{N} c_{j} e_{1}\left(\boldsymbol{x}-\boldsymbol{y}_{j}\right),
$$

where $\boldsymbol{y}_{j} \in \mathbb{R}^{n} \backslash \bar{\Omega}$ and $\boldsymbol{c}=\left\{c_{j}\right\}_{j=1}^{N} \subset \mathbb{R}$. The coefficients are determined from the boundary data, which can be done in various ways. Clearly, the approximate solution $u_{N}$ is harmonic in a neighborhood of $\bar{\Omega}$.

In the original formulation of the method by Kupradze and Aleksidze 33, for the solution of the Dirichlet problem for Laplace's equation in a bounded domain $\Omega \subset \mathbb{R}^{n}, n=2,3$, without holes, the singularities were located on a pseudoboundary, i.e., a prescribed boundary $\partial \Omega^{\prime}$ of a domain $\Omega^{\prime}$, for which $\bar{\Omega} \subset \Omega^{\prime}$. In the same work, the MFS was also developed for problems in linear elasticity. Kupradze also suggested the MFS for time-dependent problems [31; in particular, for the solution of the heat equation. The MFS was first investigated, as a numerical technique, by Mathon and Johnston 39] in 1977. In their work, the coefficients in (1.7) were chosen to minimize the $L^{2}$-distance of the approximate solution from the boundary data of (1.5). The locations of the singularities are also to be determined during the minimization process which results in a nonlinear least-squares problem. 


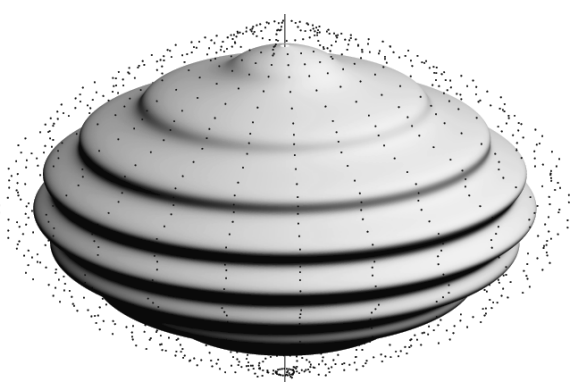

Figure 1. A typical distribution of singularities on a pseudoboundary of an axisymmetric domain

During the last four decades several formulations of the MFS have appeared in the literature. The two most popular are the following:

- In the first, the locations of the singularities are on a fixed surface. This formulation leads to a linear system.

- In the second, they are determined as part of the solution of the discrete problem. This formulation leads to a nonlinear least-squares problem.

In the first formulation, the coefficients can be obtained by collocation of the boundary data. In the case of (1.5), this is done by choosing $M$ points $\left\{\boldsymbol{y}_{j}\right\}_{j=1}^{M}$ on $\partial \Omega^{\prime}$ - the singularities - and $N$ collocation points $\left\{\boldsymbol{x}_{k}\right\}_{k=1}^{N}$ on $\partial \Omega$, and require that the approximate solution $u_{M, N}$ satisfies

$$
u_{M, N}\left(\boldsymbol{x}_{k} ; \boldsymbol{c}\right)=\sum_{j=1}^{M} c_{j} \varphi\left(\boldsymbol{x}_{k}, \boldsymbol{y}_{j}\right)=f\left(\boldsymbol{x}_{k}\right), \quad k=1, \ldots, N .
$$

This is an $M \times N$ linear system. If $M=N$, the coefficients can be determined uniquely, provided the matrix $G=\left(\varphi\left(\boldsymbol{x}_{k}, \boldsymbol{y}_{j}\right)\right)_{k, j=1}^{N}$, is nonsingular. If $M>N$, the system (1.8) is over-determined. In such a case, the coefficients can be chosen by linear least-squares, i.e., by minimizing the quantity

$$
F(\boldsymbol{c})=\frac{1}{N} \sum_{k=1}^{N}\left(u_{M, N}\left(\boldsymbol{x}_{k} ; \boldsymbol{c}\right)-f\left(\boldsymbol{x}_{k}\right)\right)^{2} \approx \frac{1}{|\partial \Omega|}\left\|u_{M, N}(\cdot, \boldsymbol{c})-f\right\|_{L^{2}(\partial \Omega)}^{2},
$$

where $|\partial \Omega|$ is the area of $\partial \Omega$. A description and analysis of the linear least-squares MFS can be found in 21, 30, 49. A weighted least-squares algorithm is developed in [45, in which the error on the boundary is minimized with respect to a suitable discrete Sobolev norm. In particular, the coefficients are chosen to minimize a quantity approximating the distance $\left\|u_{M, N}(\cdot, \boldsymbol{c})-f\right\|_{H^{s}(\partial \Omega)}$, for suitable values of $s>0$. Finally, if $M<N$, then (1.8) has in general infinitely many solutions. Criteria for choosing the most appropriate approximate solution appear in [48.

There is considerable literature concerning error estimates, stability and convergence analyses of the MFS for boundary value problems in specific domains with specific distributions of the singularities and collocation points. In the case of the Dirichlet problem for Laplace's equation in $D_{\varrho}$, the disk of radius $\varrho$, and with the circumference of a concentric disk as a pseudo-boundary, it is shown that the supremum error in the MFS approximation $\varepsilon_{N}=\sup _{\boldsymbol{x} \in \bar{D}_{e}}\left|u_{N}(\boldsymbol{x})-u(\boldsymbol{x})\right|$, tends to zero as $N$, the number of singularities and collocation points, tends to infinity, provided 
that both singularities and collocation points are uniformly distributed on $\partial \Omega$ and $\partial \Omega^{\prime}$ and the boundary data have absolutely convergent Fourier series. The rate of convergence increases as the smoothness of the data improves. In particular, if the boundary data are analytic, then the convergence is exponential, whereas if they belong to $C^{\ell}\left(\partial D_{\varrho}\right)$, then the error is $\mathcal{O}\left(N^{-\ell+1}\right)$. (See [27, 45].) In 28], this result is generalized to regions in the plane whose boundaries are analytic Jordan curves, while in [55, the same result is generalized to annular regions. Convergence of the MFS for the Helmholtz equation in the exterior of a disk was established in [56].

As reported by several authors, in the above formulation there are two contradictory facts:

A. The approximation improves as the distance between the pseudo-boundary and the boundary increases. In particular, it is shown that the MFS approximation of the Dirichlet problem in a disk $D_{\varrho}$ and pseudo-boundary a concentric circumference of radius $R>\varrho$, converges exponentially to the solution with $N$ and $\varrho / R$. More precisely, it is shown that [50]

$$
\sup _{\boldsymbol{x} \in \bar{D}_{\varrho}}\left|u_{N}(\boldsymbol{x})-u(\boldsymbol{x})\right|=\mathcal{O}\left(\left(\frac{\varrho}{R}\right)^{N / 2}\right),
$$

provided that there exists a harmonic extension of $u$ in a larger disk.

B. The condition number $\kappa=\|G\| \cdot\left\|G^{-1}\right\|$ of the coefficient matrix $G=$ $\left(\varphi\left(\boldsymbol{x}_{k}, \boldsymbol{y}_{\ell}\right)\right)$ grows exponentially with $N$ and $R / \varrho$. In fact, Kitagawa 29. (see also [50]) has shown that

$$
\kappa \sim \frac{\log R}{2} N\left(\frac{R}{\varrho}\right)^{N / 2}
$$

The poor conditioning of the MFS is widely reported in the literature (see, for example, [21]). In particular, Aleksidze [3] demonstrated that the matrices which orthonormalize the fundamental solutions, in the sense of the Hilbert space $L^{2}(\Omega)$, become extremely ill-conditioned, as the number of fundamental solutions increases.

The poor conditioning of the MFS can be alleviated, either by preconditioning of the system matrix $G$ (see, for example, [51] and references therein) or by iterative refinement (see [11]). This ill-conditioning can be removed in special cases where an accurate diagonalization of the system matrix is possible (see [45]).

Despite these shortcomings, the MFS remains a popular meshless technique for the solution of elliptic boundary value problems in which the fundamental solution of the underlying partial differential equation is known, for the following reasons:

- its simplicity and the ease with which it can be implemented;

- unlike the boundary element method, it does not require an elaborate discretization of the boundary;

- it does not involve potentially troublesome and costly integrations over the boundary;

- it can be applied even in the case of domains with irregular boundaries;

- it requires little data preparation;

- the evaluation of the approximate solution at a point in the interior of the domain can be carried out directly unlike the boundary element method for which a quadrature is needed;

- the derivatives of the MFS approximation can also be evaluated directly. 
For further details see [16]. Comprehensive reference lists of applications of the MFS can be found in [13, 14, 17, 22]. Finally, a mathematical foundation of the MFS for the numerical solution of a variety of boundary value problems in mathematical physics can be found in [4].

In this work, we investigate the applicability of the MFS in various elliptic problems. More specifically, we provide answers to the question:

Let $\mathcal{L}$ be an elliptic operator and $\varphi$ a fundamental solution of $\mathcal{L}$. Is the space $\mathcal{X}$ of linear combinations of the form (1.7), where the $\boldsymbol{y}_{j}$ 's lie on a prescribed pseudoboundary, dense in the space $\mathcal{Y}$ of all solutions of the equation $\mathcal{L} u=0$ in $\Omega$ ?

We provide extensions to the results of Kupradze and Aleksidze [33, and of Bogomolny [9. In particular, Kupradze and Aleksidze proved that if $\Omega$ and $\Omega^{\prime}$ are sufficiently smooth bounded domains in $\mathbb{R}^{3}$, without holes, with $\bar{\Omega} \subset \Omega^{\prime}$, then linear combinations of the fundamental solutions of the Laplacian in $\mathbb{R}^{3}$ with singularities on $\partial \Omega^{\prime}$ are dense in $L^{2}(\partial \Omega)$ with respect to the $L^{2}$-norm. They also obtained similar density results for the Cauchy-Navier system in linear elasticity. Bogomolny obtained density of $\mathcal{X}$ for harmonic and biharmonic problems with respect to the norm of the Sobolev space $H^{s}(\Omega)$, when $\Omega$ is a bounded domain possessing a smooth boundary. In his proof, Bogomolny used the duality argument (an application of the Hahn-Banach Theorem) of the proo 2 of Theorem 3 in Browder 10.

In our work, we study approximation by fundamental solutions with respect to more pertinent norms, namely the norms of the spaces $C^{\ell}(\bar{\Omega})$. Such norms correspond to the classical formulations of elliptic boundary value problems. Analogous density results are obtainable with respect to the norms of the spaces of uniformly Hölder continuous functions $C^{\ell, \sigma}(\bar{\Omega})$. Our domains may possess holes 3 . More specifically, the complements of our domains are not required to be connected. We obtain our density results assuming a rather weak boundary regularity requirement, namely the segment condition. This is the most important element of originality of this work, since all previous density results were obtained for domains with smooth boundaries, while most numerical applications were for irregular geometries (i.e., domains with corners and/or cusps). In this work, we are mainly concerned with the applicability of the MFS to problems of physical interest. We restrict our attention to partial differential operators - scalar and systems — with constant coefficients and known fundamental solutions.

The paper is organized as follows:

Section 2. We define the notion of the embracing pseudo-boundary and the segment condition. Next, we briefly describe the function spaces which are used in

\footnotetext{
${ }^{2}$ In [10], cf., Theorem 3, F. Browder proved the following result:

Browder's Theorem. Let $\mathcal{L}$ be a linear operator with coefficients in $C^{\infty}(G)$, where $G$ is a domain in $\mathbb{R}^{n}$ without holes. Assume that both $\mathcal{L}$ and $\mathcal{L}^{\star}$ satisfy condition $(\mathrm{U})_{s}$ and there exists a bi-regular fundamental solution e of $\mathcal{L}$ satisfying $\mathcal{L}_{\boldsymbol{x}} e(\cdot, \boldsymbol{y})=\delta_{\boldsymbol{y}}$ and $\mathcal{L}_{\boldsymbol{y}}^{\star} e(\boldsymbol{x}, \cdot)=\delta_{\boldsymbol{x}}$, for every $\boldsymbol{x}, \boldsymbol{y} \in G$. Let $\Omega$ be an open subset of $G$, satisfying the cone condition, such that $\bar{\Omega} \subset G$ and $G \backslash \bar{\Omega}$ does not contain any closed connected components. Let $V$ be an open subset of $G$, such that $\bar{\Omega} \cap \bar{V}=\varnothing$. Then every solution $u$ of $\mathcal{L} u=0$ in $\Omega$, which lies in $C^{\infty}(\Omega) \cap C(\bar{\Omega})$ can be approximated, with respect to the uniform norm, by finite linear combinations of functions of the form $e(\cdot, \boldsymbol{y})$, where $\boldsymbol{y} \in V$.

${ }^{3}$ Very often in the literature the domains without (resp., with) holes are called simply (resp., multiply) connected. While this is correct for two-dimensional domains, simply connected domains in $\mathbb{R}^{n}, n>2$, may possess holes. In this work, we say that a domain does not possess holes if and only if its complement is connected.
} 
this paper. Finally, we state Lemma 1) which is used in the proofs of our density results.

Section 3. We investigate the applicability of the MFS for harmonic problems in bounded domains. We establish a $C^{\ell}$-density result for $n$-dimensional problems, where $n \geq 3$. In the two-dimensional case, linear combinations of fundamental solutions with singularities on a prescribed pseudo-boundary are not always dense in the space of harmonic functions, and alternative formulations of the method are proposed. In particular, it is proved (when $n=2$ ) that the required density result holds if the pseudo-boundary is a subset of a unit disk; this allows the use of rescaled fundamental solutions (i.e., $e_{1}^{R}(\boldsymbol{x})=e_{1}\left(R^{-1} \boldsymbol{x}\right)$, where $R$ is sufficiently large) in the MFS approximation. In the case of an analytic pseudo-boundary $\partial \Omega^{\prime}$, we show that the same density results are obtained even if the locations of the singularities are restricted to an open subset of $\partial \Omega^{\prime}$.

Section 4. We investigate similar questions for the biharmonic and more generally the $m$-harmonic (i.e., $\mathcal{L}=(-\Delta)^{m}$ ) operators. We provide an example where linear combinations of fundamental solutions of the biharmonic operator, with singularities located on a given pseudo-boundary, do not approximate all biharmonic functions. Density of such linear combinations is achieved when we include, in the linear combinations of the fundamental solutions of $\Delta^{2}$, linear combinations of the fundamental solutions of $-\Delta$. Analogous density results hold for the solutions of the $m$-harmonic equation. We also study an alternative MFS formulation which exploits Almansi's representation.

Section 5. Similar density results are obtained for the fundamental solution of the modified Helmholtz operator: $\mathcal{L}=\Delta-\kappa^{2}$, where $\kappa>0$. We also investigate the applicability of the MFS for operators of the form: $\mathcal{L}=\prod_{j=1}^{m}\left(\Delta-\kappa_{j}^{2}\right)^{\nu_{j}}$, where $\left\{\kappa_{j}\right\}_{j=1}^{m}$ are distinct nonnegative reals and $\left\{\nu_{j}\right\}_{j=1}^{m}$, positive integers.

Section [6. We describe how the MFS is formulated for homogeneous systems of partial differential equations and provide similar density results for the CauchyNavier system of elastostatics in three space dimensions. Analogous density results are provided for a $4 \times 4$ system which describes a linear model in the static theory of thermo-elasticity in three space dimensions.

Section 7 We provide a summary of this work and concluding remarks and ideas for possible extensions.

Appendix. In order to avoid overloading the main text, the proof of Lemma 1 is given in the Appendix.

\section{A FEW WORDS ON FUNCTION SPACES AND BOUNDARIES}

2.1. Boundary regularity requirements. The density results in 9,33 require domains with smooth boundaries. Nevertheless, most numerical applications of the MFS are for domains with nonsmooth boundaries. In the present work our domains are required to satisfy the following condition:

Definition 1 (The Segment Condition). Let $\Omega$ be an open subset of $\mathbb{R}^{n}$. We say that $\Omega$ satisfies the segment condition if for every $\boldsymbol{x} \in \partial \Omega$ there is a neighborhood $U_{\boldsymbol{x}}$ of $\boldsymbol{x}$ and a nonzero vector $\boldsymbol{\xi}_{\boldsymbol{x}}$ in $\mathbb{R}^{n}$, such that, if $\boldsymbol{y} \in U_{\boldsymbol{x}} \cap \bar{\Omega}$, then $\boldsymbol{y}+t \boldsymbol{\xi}_{\boldsymbol{x}} \in \Omega$, for every $t \in(0,1)$. 


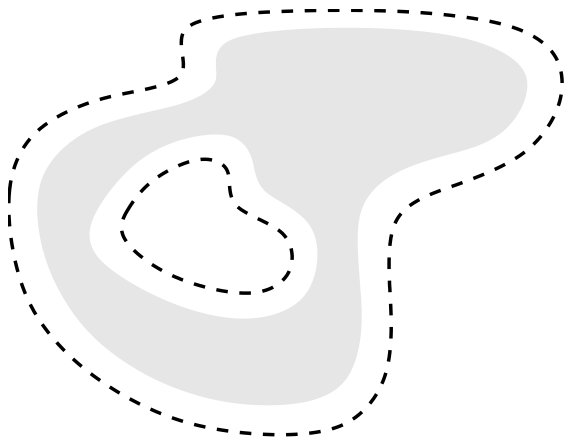

(a) The unbounded component of $\mathbb{R}^{n} \backslash \bar{\Omega}$ does not contain a bounded component of $\mathbb{R}^{n} \backslash \bar{\Omega}^{\prime}$.

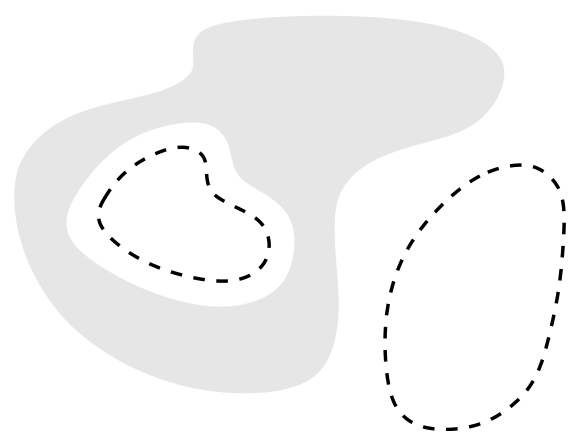

(b) The unbounded component of $\mathbb{R}^{n} \backslash \bar{\Omega}$ contains a bounded component of $\mathbb{R}^{n} \backslash \bar{\Omega}^{\prime}$.

Figure 2. In each figure, the gray region is the domain $\Omega$; the broken lines correspond to the embracing pseudo-boundary $\partial \Omega^{\prime}$.

Note that the segment condition in weaker than the cone condition 4 and allows the boundaries to have corners and cusps. Note also that $\Omega$ satisfies the segment condition if and only if $\mathbb{R}^{n} \backslash \bar{\Omega}$ does. Also, the boundaries of domains satisfying this condition are $(n-1)$-dimensional and their measure is zero. However, if a domain satisfies the segment condition it cannot lie on both sides of any part of its boundary (2]). It is not hard to prove that, if a domain satisfies the segment condition, then every connected component of its complement has a nonempty interior. In fact, domains satisfying the segment condition coincide with the interior of their closure. Finally, bounded domains satisfying the segment condition can have only finitely many holes (i.e., their complement can have finitely many connected components).

2.2. The embracing pseudo-boundary. In our density results, the singularities of the fundamental solutions are located on a prescribed pseudo-boundary, i.e., the boundary $\partial \Omega^{\prime}$ of a domain $\Omega^{\prime}$ embracing $\Omega$ (see Figure 2).

Definition 3. Let $\Omega, \Omega^{\prime}$ be open connected subsets of $\mathbb{R}^{n}$. We say that $\Omega^{\prime}$ embraces $\Omega$ if $\bar{\Omega} \subset \Omega^{\prime}$, and for every connected component $V$ of $\mathbb{R}^{n} \backslash \bar{\Omega}$, there is an open connected component $V^{\prime}$ of $\mathbb{R}^{n} \backslash \bar{\Omega}^{\prime}$ such that $\bar{V}^{\prime} \subset V$.

For example, the annulus $A_{r_{1}, r_{2}}=\left\{\boldsymbol{x} \in \mathbb{R}^{2}: r_{1}<|\boldsymbol{x}|<r_{2}\right\}$ embraces the annulus $A_{\varrho_{1}, \varrho_{2}}$ provided that $0<r_{1}<\varrho_{1}<\varrho_{2}<r_{2}$. On the other hand, a disk cannot embrace an annulus. Note that, if $\Omega$ does not have holes and $U \neq \varnothing$ is open, such that $\bar{\Omega} \cap \bar{U}=\varnothing$, then $\mathbb{R}^{n} \backslash \bar{U}$ embraces $\Omega$. In fact, if $\Omega$ is bounded and embraced by $\Omega^{\prime}$, then $\Omega$ can have only finitely many holes.

\footnotetext{
${ }^{4}$ Certain Sobolev Imbedding and Extension Theorems require the following boundary requirement:

Definition 2 (The Cone Condition). Let $\Omega$ be an open subset of $\mathbb{R}^{n}$. We say $\Omega$ satisfies the cone condition if there is a finite open cover $\left\{U_{j}\right\}_{j=1}^{J}$ of $\partial \Omega$ and an $h>0$, such that, for every $x \in \Omega \cup U_{j}$, there is a unit vector $\boldsymbol{\xi}_{j} \in \mathbb{R}^{n}$ such that the cone

$$
C_{h}\left(\boldsymbol{\xi}_{j}\right)=\left\{\boldsymbol{y}=\boldsymbol{x}+r \boldsymbol{\xi}: r \in(0, h) \text { and }\left|\boldsymbol{\xi}-\boldsymbol{\xi}_{j}\right|<h\right\},
$$

is a subset of $\Omega$.
} 
An alternative definition of the embracing pseudo-boundary is the following.

Definition $3^{\prime}$. Let $\Omega, \Omega^{\prime}$ be open connected subsets of $\mathbb{R}^{n}$. We say that $\Omega^{\prime}$ embraces $\Omega$ if $\bar{\Omega} \subset \Omega^{\prime}$ and $\Omega^{\prime} \backslash \Omega$ does not contain any closed connected components.

If both $\Omega$ and $\Omega^{\prime}$ satisfy the segment condition, then Definition 3 implies Definition 3

\subsection{Our function spaces.}

2.3.1. The spaces $C^{\ell}(\bar{\Omega})$ and $C^{\ell, \sigma}(\bar{\Omega})$. If $\Omega$ is an open domain in $\mathbb{R}^{n}$, then the space $C^{\ell}(\Omega)$, where $\ell$ is a nonnegative integer, contains all functions $u$ which, together with all their partial derivatives $D^{\alpha} u$ of orders $|\alpha| \leq \ell$, are continuous in $\Omega$ and $C^{\infty}(\Omega)=\bigcap_{\ell \in \mathbb{N}} C^{\ell}(\Omega)$. The space $C^{\ell}(\bar{\Omega})$ consists of all functions $u \in C^{\ell}(\Omega)$ for which $D^{\alpha} u$ is uniformly continuous and bounded in $\Omega$ for all $|\alpha| \leq \ell$. In fact, $C^{\ell}(\bar{\Omega})$ is a Banach space with norm

$$
|u|_{\ell, \bar{\Omega}}=\max _{|\alpha| \leq \ell} \sup _{\boldsymbol{x} \in \bar{\Omega}}\left|D^{\alpha} u(\boldsymbol{x})\right| .
$$

If $\Omega$ is bounded, then the elements of the dual of $C(\bar{\Omega})$ are represented by the signed Borel measures on $\bar{\Omega}$. The elements of the dual of $C^{\ell}(\bar{\Omega})$ can also be represented by signed Borel measures. For every $\nu \in\left(C^{\ell}(\bar{\Omega})\right)^{\prime}$, there exist $\left\{\nu_{\alpha}\right\}_{|\alpha| \leq \ell} \subset \mathfrak{M}(\bar{\Omega})$ such that

$$
\nu(u)=\sum_{|\alpha| \leq \ell} \int_{\bar{\Omega}} D^{\alpha} u d \nu_{\alpha} \quad \text { for every } \quad u \in C^{\ell}(\bar{\Omega}) .
$$

Also, $C_{0}^{\ell}\left(\mathbb{R}^{n}\right)$ is the set of functions $u$ which, together with all their partial derivatives $D^{\alpha} u$ of orders $|\alpha| \leq \ell$, are continuous in $\mathbb{R}^{n}$ and satisfy $\lim _{|\boldsymbol{x}| \rightarrow \infty} D^{\alpha} u(\boldsymbol{x})=0$, for every $|\alpha| \leq \ell$. Clearly, $C_{0}^{\ell}\left(\mathbb{R}^{n}\right)$ is a Banach space with norm $|u|_{\ell, \mathbb{R}^{n}}=$ $\max _{|\alpha| \leq \ell} \sup _{\boldsymbol{x} \in \mathbb{R}^{n}}\left|D^{\alpha} u(\boldsymbol{x})\right|$. The elements of $\left(C^{\ell}(\bar{\Omega})\right)^{\prime}$ define bounded linear functionals on $C_{0}^{\ell}\left(\mathbb{R}^{n}\right)$.

Now let $\sigma \in(0,1)$ and let $\Omega$ be an open bounded domain in $\mathbb{R}^{n}$. The space of Hölder functions $\Lambda^{0, \sigma}(\bar{\Omega})$ consists of all functions $u$, such that $[u]_{\sigma}=\sup _{\delta>0} \omega_{\sigma}(u, \delta)$ $<\infty$, where

$$
\omega_{\sigma}(u, \delta)=\sup _{\substack{\boldsymbol{x}, \boldsymbol{y} \in \bar{\Omega} \\ 0<|\boldsymbol{x}-\boldsymbol{y}|<\delta}} \frac{|u(\boldsymbol{x})-u(\boldsymbol{y})|}{|\boldsymbol{x}-\boldsymbol{y}|^{\sigma}} .
$$

The space $\Lambda^{0, \sigma}(\bar{\Omega})$ is a Banach space with norm $|u|_{0, \sigma}=|u|_{0}+[u]_{\sigma}$, where $|\cdot|_{0}$ is the norm of $C(\bar{\Omega})$. In general, if $k \in \mathbb{N}$, then the space $\Lambda^{k, \sigma}(\bar{\Omega})$ consists of all functions $u$ which, together with all their partial derivatives $D^{\alpha} u,|\alpha| \leq k$, belong to $C^{0, \sigma}(\bar{\Omega})$. The space $\Lambda^{k, \sigma}(\bar{\Omega})$ is a Banach space with respect to the norm given by $|u|_{k, \sigma}=|u|_{k}+\max _{|\alpha| \leq k}\left[D^{\alpha} u\right]_{\sigma}$. If $u \in \Lambda^{k, \sigma}(\bar{\Omega})$ and $\lim _{\delta \rightarrow 0} \omega_{\delta}(u, \delta)=0$, then $u$ is called uniformly Hölder continuous of order $(k, \sigma)$ in $\bar{\Omega}$. The set of uniformly Hölder continuous of order $(k, \sigma)$ in $\bar{\Omega}$, which is denoted by $C^{k, \sigma}(\bar{\Omega})$, is a closed

\footnotetext{
${ }^{5}$ This representation can be achieved by the isometric imbedding $\mathcal{P}: C^{\ell}(\bar{\Omega}) \rightarrow C\left(\bar{\Omega}^{(\ell)}\right)$, where $\bar{\Omega}^{(\ell)}$ is the union of mutually disjoint copies $\left\{\bar{\Omega}_{\alpha}\right\}_{|\alpha| \leq \ell}$ of $\bar{\Omega}$ and $\mathcal{P} u=\left(D^{\alpha} u\right)_{|\alpha| \leq \ell}$. The dual of $C\left(\bar{\Omega}^{(\ell)}\right)$ is representable by a sum of signed Borel measures $\left\{\nu_{\alpha}\right\}_{|\alpha| \leq m}$, with $\operatorname{supp} \nu_{\alpha} \subset \bar{\Omega}_{\alpha}$, and since $C=\mathcal{P}\left[C^{\ell}(\bar{\Omega})\right]$ is a closed subspace of $C^{\ell}(\bar{\Omega})$, every bounded linear functional on $C$ can be extended to a bounded linear functional $\nu$ on $C^{\ell}(\bar{\Omega})$, due to the Hahn-Banach theorem, and thus $\nu$ can be expressed in the form (2.1).
} 
subspace of $\Lambda^{k, \sigma}(\bar{\Omega})$ and thus a Banach space as well. Clearly, if $u \in \Lambda^{k, \sigma}(\bar{\Omega})$ can be approximated in the $|\cdot|_{k, \sigma}$-norm by functions which are $C^{\infty}$ in a neighborhood of $\bar{\Omega}$, then $u \in C^{k, \sigma}(\bar{\Omega})$. We extend for $\sigma=0$ the definition of the space $C^{k, \sigma}(\bar{\Omega})$ by setting $C^{k, 0}(\bar{\Omega})=C^{k}(\bar{\Omega})$.

2.3.2. Test functions and distributions. By $\mathscr{D}(\Omega)$ or $C_{0}^{\infty}(\Omega)$ we denote the space of infinitely many times differentiable functions with compact support in $\Omega$. The elements of $\mathscr{D}(\Omega)$ are known as the test functions. The space of test functions $\mathscr{D}(\Omega)$ is a Fréchet space, i.e., its topology is induced by a complete invariant metric. (See [42, Chapter 6].) A sequence $\left\{\psi_{n}\right\}_{n \in \mathbb{N}} \subset \mathscr{D}(\Omega)$ converges to zero, with respect to the topology of $\mathscr{D}(\Omega)$, if there exists a compact subset $K$ of $\Omega$ such that $\operatorname{supp} \psi_{n} \subset$ $K$, for all $n \in N$, and $D^{\alpha} \psi_{n} \rightarrow 0$, uniformly in $K$, for every multi-index $\alpha \in \mathbb{N}^{n}$. The space $\mathscr{D}(\Omega)$ possesses a topological dual $\mathscr{D}^{\prime}(\Omega)$, the elements of which are the continuous linear functionals on $\mathscr{D}(\Omega)$ known as the distributions on $\Omega$. We shall be denoting by $\langle\cdot, \cdot\rangle$ the pairing between $\mathscr{D}$ and $\mathscr{D}^{\prime}$. Any function $f \in L_{\mathrm{loc}}^{1}(\Omega)$ defines a distribution $T_{f}$ through the pairing

$$
\left\langle\psi, T_{f}\right\rangle=\langle\psi, f\rangle=\int_{\Omega} \psi f d \boldsymbol{x}, \quad \psi \in \mathscr{D}(\Omega) .
$$

If $f$ is sufficiently smooth, then $T_{D^{\alpha} f}(\psi)=\int_{\Omega} \psi D^{\alpha} f d \boldsymbol{x}=(-1)^{|\alpha|} \int_{\Omega} D^{\alpha} \psi f d \boldsymbol{x}$. Nevertheless, the expression $(-1)^{|\alpha|} \int_{\Omega} D^{\alpha} \psi f d \boldsymbol{x}$ defines a distribution, even if $f$ is not smooth. In fact, if $T$ is a distribution, then so is $D^{\alpha} T$ defined accordingly as $\left\langle\psi, D^{\alpha} T\right\rangle=(-1)^{|\alpha|}\left\langle D^{\alpha} \psi, T\right\rangle$. Similarly, if $\mathcal{L}$ is a differential operator with constant coefficients, then $\mathcal{L} T$ is defined by $\langle\psi, \mathcal{L} T\rangle=\left\langle\mathcal{L}^{\star} \psi, T\right\rangle$, and it is also a distribution. We say that a distribution $T \in \mathscr{D}^{\prime}(\Omega)$ has compact support if there exists a compact set $K \subset \Omega$, such that, if $\left.\psi\right|_{K}=0$, then $T(\psi)=0$. If $u \in \mathscr{D}^{\prime}\left(\mathbb{R}^{n}\right)$ and $\psi \in \mathscr{D}\left(\mathbb{R}^{n}\right)$, then their convolution $u * \psi$ is defined as in the case in which $u$ is a locally integrable function, namely,

$$
(u * \psi)(\boldsymbol{x})=\int_{\mathbb{R}^{n}} \psi(\boldsymbol{x}-\boldsymbol{y}) u(\boldsymbol{y}) d \boldsymbol{y}=\left\langle\tau_{-\boldsymbol{x}} \check{\psi}, u\right\rangle,
$$

where $\tau_{\boldsymbol{x}} \psi(\boldsymbol{y})=\psi(\boldsymbol{x}-\boldsymbol{y})$ and $\check{\psi}(\boldsymbol{x})=\psi(-\boldsymbol{x})$. Clearly, $u * \psi$ defines a function in $C^{\infty}\left(\mathbb{R}^{n}\right)$, and if $u$ has compact support, then $u * \psi \in \mathscr{D}\left(\mathbb{R}^{n}\right)$. Finally, if $u, v \in$ $\mathscr{D}^{\prime}\left(\mathbb{R}^{n}\right)$, one of which (say $v$ ) has compact support, then their convolution is defined as expected $\langle\psi, u * v\rangle=\langle\breve{v} * \psi, u\rangle$. In fact, $u * v \in \mathscr{D}^{\prime}\left(\mathbb{R}^{n}\right)$. It is noteworthy that, if $e$ is a fundamental solution of the partial differential operator $\mathcal{L}$ and $T \in \mathscr{D}^{\prime}(\Omega)$ with compact support, then we have

$$
\mathcal{L}(e * T)=(\mathcal{L} e) * T=\delta * T=T,
$$

in the sense of distributions, where $\langle\psi, \delta\rangle=\psi(0)$.

2.3.3. The main lemma. Our density results are based on the following lemma:

Lemma 1. Let $\mathcal{L}=\sum_{|\alpha| \leq m} a_{\alpha} D^{\alpha}$ be an elliptic operator with constant coefficients in $\mathbb{R}^{n}$ and $e=e(\boldsymbol{x})$ a fundamental solution of $\mathcal{L}$. Also, let $\Omega$ be an open bounded subset of $\mathbb{R}^{n}$ satisfying the segment condition and $\nu \in\left(C^{\ell}(\bar{\Omega})\right)^{\prime}$. If $\vartheta=e * \nu$ is the convolution of the distributions $e$ and $\nu$ and if $\operatorname{supp} \vartheta \subset \bar{\Omega}$, then there exists a sequence $\left\{\vartheta^{k}\right\}_{k \in \mathbb{N}} \subset \mathscr{D}^{\prime}\left(\mathbb{R}^{n}\right)$ with $\operatorname{supp} \vartheta^{k} \subset \Omega$ and $\left\{\mathcal{L} \vartheta^{k}\right\}_{k \in \mathbb{N}} \subset\left(C^{\ell}(\bar{\Omega})\right)^{\prime}$, 
such that $\left\{\mathcal{L} \vartheta^{k}\right\}_{k \in \mathbb{N}}$ converges to $\nu$ in the weak $k^{\star}$ sense of $\left(C^{\ell}(\bar{\Omega})\right)^{\prime}$, i.e., for every $u \in C^{\ell}(\bar{\Omega})$,

$$
\lim _{k \rightarrow \infty} \mathcal{L} \vartheta^{k}(u)=\nu(u)
$$

Proof. See Appendix A.

\section{HARMONIC PROBLEMS}

3.1. A density result. Our first density result corresponds to the classical solutions of Dirichlet and Neumann problems for Laplace's equation in bounded domains.

Theorem 1. Let $\Omega, \Omega^{\prime}$ be open bounded domains in $\mathbb{R}^{n}$ with $\Omega$ satisfying the segment condition and $\Omega^{\prime}$ embracing $\Omega$ and let $\ell$ be a nonnegative integer. Then the space $\mathcal{X}$ of finite linear combinations of the form $\sum_{j=1}^{N} c_{j} e_{1}\left(\boldsymbol{x}-\boldsymbol{y}_{j}\right)$, where $e_{1}$ is given by (1.6) and $\left\{\boldsymbol{y}_{j}\right\}_{j=1}^{N} \subset \partial \Omega^{\prime}$, is dense in

$$
\mathcal{Y}_{\ell}=\left\{u \in C^{2}(\Omega): \Delta u=0 \text { in } \Omega\right\} \cap C^{\ell}(\bar{\Omega}),
$$

with respect to the norm of the space $C^{\ell}(\bar{\Omega})$ if $n \geq 3$. If $n=2$, then the linear sum $\mathcal{X} \oplus\left\{\left.c \cdot 1\right|_{\Omega}: c \in \mathbb{R}\right\}$ is dense in $\mathcal{Y}_{\ell}$ also with respect to the same norm.

Proof. We follow the ideas developed in [9] and [10]. Both sets $\mathcal{X}$ and $\mathcal{Y}_{\ell}$ are linear subspaces of $C^{\ell}(\bar{\Omega})$. If $\nu \in\left(C^{\ell}(\bar{\Omega})\right)^{\prime}$, then there exist $\left\{\nu_{\alpha}\right\}_{|\alpha| \leq \ell} \subset \mathfrak{M}(\bar{\Omega})$, where $\mathfrak{M}(\bar{\Omega})$ is the set of signed Borel measures on $\bar{\Omega}$, such that

$$
\nu(u)=\sum_{|\alpha| \leq \ell} \int_{\bar{\Omega}} D^{\alpha} u d \nu_{\alpha} \quad \text { for every } \quad u \in C^{\ell}(\bar{\Omega}) .
$$

From the Hahn-Banach theorem, it suffices to show that $\mathcal{X}^{\perp} \subset \mathcal{Y}_{\ell}^{\perp}$, i.e.,

$$
\text { if } \quad \nu \in\left(C^{\ell}(\bar{\Omega})\right)^{\prime} \quad \text { and } \begin{gathered}
\nu(u)=0 \\
\text { for every } u \in \mathcal{X}
\end{gathered} \text { then } \begin{gathered}
\nu(u)=0 . \\
\text { for every } u \in \mathcal{Y}_{\ell}
\end{gathered}
$$

Let $\nu \in\left(C^{\ell}(\bar{\Omega})\right)^{\prime}$ be such that $\nu(u)=0$, for every $u \in \mathcal{X}$. In particular, if $\boldsymbol{x} \in \partial \Omega^{\prime}$, then the function $u(\boldsymbol{y})=e_{1}(\boldsymbol{y}-\boldsymbol{x})=\tau_{\boldsymbol{x}} e_{1}(\boldsymbol{y})$ belongs to $\mathcal{X}$ and

$$
0=\nu(u)=\left\langle\nu\left(\tau_{\boldsymbol{x}} e_{1}\right)=\left(e_{1} * \nu\right)(\boldsymbol{x}) .\right.
$$

Thus the convolution $\vartheta=e_{1} * \nu$ vanishes on $\partial \Omega^{\prime}$. Note that $\vartheta$ defines a distribution in $\mathbb{R}^{n}$, as a convolution of two distributions, one of which (namely $\nu$ ) is of compact support (i.e., $\operatorname{supp} \nu \subset \bar{\Omega}$ ). Meanwhile, $\vartheta$ is a real analytic function, and in fact, a harmonic function in $\mathbb{R}^{n} \backslash \bar{\Omega}$. Also, $-\Delta \vartheta=\nu$, in the sense of distributions in $\mathbb{R}^{n}$. Let $U$ be the unbounded connected component of $\mathbb{R}^{n} \backslash \bar{\Omega}$. Since $\Omega^{\prime}$ embraces $\Omega$, there is a connected component $U^{\prime}$ of $\mathbb{R}^{n} \backslash \bar{\Omega}^{\prime}$ such that $\bar{U}^{\prime} \subset U$. Clearly, $\partial U^{\prime} \subset \partial \Omega^{\prime}$, and therefore $\vartheta$ vanishes on $\partial U^{\prime}$. If $U^{\prime}$ is bounded, then $\vartheta$ vanishes in $U^{\prime}$, from the maximum principle. Consequently, $\vartheta$ vanishes in the whole of $U$, being a real analytic function. If $U^{\prime}$ is unbounded, then for $\boldsymbol{x} \in U^{\prime}$ we have

$$
\vartheta(\boldsymbol{x})=\nu\left(\tau_{\boldsymbol{x}} e_{1}\right)=\sum_{|\alpha| \leq \ell} \int_{\bar{\Omega}} D_{\boldsymbol{y}}^{\alpha} e_{1}(\boldsymbol{y}-\boldsymbol{x}) d \nu_{\alpha}(\boldsymbol{y}),
$$

and thus

$$
|\vartheta(\boldsymbol{x})| \leq\left(\sum_{|\alpha| \leq \ell}\left\|\nu_{\alpha}\right\|\right) \cdot\left(\sup _{\boldsymbol{y} \in \bar{\Omega},|\alpha| \leq \ell}\left|D^{\alpha} e_{1}(\boldsymbol{y}-\boldsymbol{x})\right|\right) .
$$


It is not hard to show that for $\boldsymbol{x}$ large (and $\boldsymbol{y}$ in $\bar{\Omega}$ ), we have $D^{\alpha} e_{1}(\boldsymbol{y}-\boldsymbol{x})=$ $\mathcal{O}\left(|\boldsymbol{x}|^{2-n-|\alpha|}\right)$, which combined with (3.2) provides that $\vartheta(\boldsymbol{x})=\mathcal{O}\left(|\boldsymbol{x}|^{2-n}\right)$. Therefore, $\lim _{\boldsymbol{x} \rightarrow \infty} \vartheta(\boldsymbol{x})=0$. Since $\vartheta$ vanishes also on $\partial U^{\prime}$ and is arbitrarily small on $S_{R}=\left\{\boldsymbol{x} \in \mathbb{R}^{n}:|\boldsymbol{x}|=R\right\}$, for $R$ sufficiently large, then by the maximum principle, $\vartheta$ vanishes in the whole of $U^{\prime}$. Thus $\vartheta$ vanishes in the whole of $U$, since $\vartheta$ vanishes in $U^{\prime}$, a nonempty open subset of $U$. If $U$ is a bounded component of $\mathbb{R}^{n} \backslash \bar{\Omega}$, then according to Definition 3, there is an open component $U^{\prime}$ of $\mathbb{R}^{n} \backslash \bar{\Omega}^{\prime}$ such that $\bar{U}^{\prime} \subset U$. In particular, $\partial U^{\prime} \subset \partial \Omega^{\prime}$ and thus $\vartheta$ vanishes in $\partial U^{\prime}$. Therefore, $\vartheta$ vanishes in the whole of $U^{\prime}$, and, since $\vartheta$ is harmonic in $U$, it has to vanish in the whole of $U$. Consequently, $\vartheta$ vanishes in $\mathbb{R}^{n} \backslash \bar{\Omega}$, and thus $\operatorname{supp} \vartheta \subset \bar{\Omega}$.

Let $u \in \mathcal{Y}_{\ell}$. Then if in Lemma 1 we set $\mathcal{L}=-\Delta$ and $e=e_{1}$, given by (1.6), then we obtain a sequence of distributions $\left\{\vartheta^{k}\right\}_{k \in \mathbb{N}}$ supported in $\Omega$ with $\left\{\Delta \vartheta^{k}\right\}_{k \in \mathbb{N}} \subset\left(C^{\ell}(\bar{\Omega})\right)^{\prime}$, such that

$$
\nu(u)=-\lim _{k \rightarrow \infty} \Delta \vartheta^{k}(u) .
$$

It suffices to show that $\Delta \vartheta^{k}(u)=0$. Since $\operatorname{supp} \vartheta^{k} \subset \Omega$, then we can find a $\psi \in \mathscr{D}(\Omega)$, which is equal to one in a neighborhood of $\bar{\Omega}$.

The distribution $-\Delta \vartheta^{k}$ is representable by signed Borel measures $\left\{\nu_{\alpha}^{k}\right\}_{|\alpha| \leq \ell}$, since it belongs to the dual of $C^{\ell}(\bar{\Omega})$, and in particular we have that

$$
\begin{aligned}
-\Delta \vartheta^{k}(u) & =\sum_{|\alpha| \leq \ell} \int_{\bar{\Omega}} D^{\alpha} u d \nu_{a}^{k}=\sum_{|\alpha| \leq \ell} \int_{\operatorname{supp} \vartheta^{k}} D^{\alpha} u d \nu_{a}^{k}=\sum_{|\alpha| \leq \ell} \int_{\operatorname{supp} \vartheta^{k}} D^{\alpha}(\varphi u) d \nu_{a}^{k} \\
& =-\Delta \vartheta^{k}(\varphi u)=-\left\langle\varphi u, \Delta \vartheta^{k}\right\rangle,
\end{aligned}
$$

since the functions $u$ and $\varphi u$ agree in a neighborhood of $\operatorname{supp} \vartheta^{k}$, and so do their partial derivatives of orders up to $\ell$. The last equality is due to the fact that $\varphi u$ belongs to $C^{\ell}(\bar{\Omega})$ and $\mathscr{D}(\Omega)$, and $-\Delta \vartheta^{k}$ belongs to $\left(C^{\ell}(\bar{\Omega})\right)^{\prime}$ and $\mathscr{D}^{\prime}(\Omega)$, and the two pairings coincide in the intersections of the corresponding spaces. According to the definition of the distribution $\Delta \vartheta^{k}$ we have

$$
\left\langle\varphi u, \Delta \vartheta^{k}\right\rangle=\left\langle\Delta(\varphi u), \vartheta^{k}\right\rangle .
$$

The right-hand side in the above equality is equal to zero since $\Delta(\varphi u)=\Delta u=0$ in the support of $\vartheta^{k}$, which concludes the proof of Theorem 1 for $n \geq 3$.

If $n=2$, we have also assumed that the function $1_{\bar{\Omega}}$ belongs to $\mathcal{X}$. Therefore,

$$
0=\nu\left(1_{\bar{\Omega}}\right)=\sum_{|\alpha| \leq \ell} \int_{\bar{\Omega}} D^{\alpha} 1_{\bar{\Omega}} d \nu_{\alpha}=\int_{\bar{\Omega}} 1_{\bar{\Omega}} d \nu_{0}=\nu_{0}(\bar{\Omega}) .
$$

Let $V$ be the unbounded component of $\mathbb{R}^{n} \backslash \bar{\Omega}$ and $\boldsymbol{x} \in V$, then

$$
\vartheta(\boldsymbol{x})=-\frac{1}{2 \pi}\langle\log |\cdot-\boldsymbol{x}|, \nu\rangle=-\frac{1}{2 \pi}(\langle\log |\cdot-\boldsymbol{x}|, \nu\rangle-\langle\log |\boldsymbol{x}|, \nu\rangle)-\frac{1}{2 \pi}\langle\log |\boldsymbol{x}|, \nu\rangle .
$$

Clearly, $\langle\log |\boldsymbol{x}|, \nu\rangle=\log |\boldsymbol{x}|\left\langle 1_{\bar{\Omega}}, \nu\right\rangle=0$ and, for $\boldsymbol{x}$ large and $\boldsymbol{y} \in \bar{\Omega}$ we have

$$
|\log | \boldsymbol{y}-\boldsymbol{x}|-\log | \boldsymbol{x}|| \leq \log \left(1+\frac{|\boldsymbol{y}|}{|\boldsymbol{x}|}\right)=\mathcal{O}\left(\frac{1}{|\boldsymbol{x}|}\right),
$$

whereas, for $|\alpha| \geq 1$, we have

$$
D_{\boldsymbol{y}}^{\alpha}(\log |\boldsymbol{y}-\boldsymbol{x}|-\log |\boldsymbol{x}|)=\mathcal{O}\left(|\boldsymbol{x}|^{-|\alpha|}\right) .
$$

Therefore, $\lim _{|\boldsymbol{x}| \rightarrow \infty} \vartheta(\boldsymbol{x})=0$, which implies that $\vartheta$ vanishes in $V$. 
Remark 3.1. If $\mathcal{L}=-\sum_{k, \ell=1}^{n} a_{k, \ell} \partial_{k} \partial_{\ell}$ is an elliptic operator, then a fundamental solution of $\mathcal{L}$ is given by (1.6) with $|\boldsymbol{x}|$ replaced by $\left(\sum_{k, \ell=1}^{n} A_{k, \ell} x_{k} x_{\ell}\right)^{1 / 2}$, where $A_{k, \ell}$ is the cofactor of $a_{k, \ell}$ in the matrix $A=\left(a_{k, \ell}\right)_{k, \ell=n}^{n}$. Theorem 1 also holds for the operator $\mathcal{L}$.

Remark 3.2. If in Theorem 1 the space $C^{\ell}(\bar{\Omega})$ is replaced by the space $C^{\ell, \sigma}(\bar{\Omega})$, $\sigma \in(0,1)$, then the corresponding density result is still valid. For details see [47.

Remark 3.3. In Theorem 1 the requirement that the boundary of the domain $\Omega^{\prime}$ is bounded could be replaced by the weaker requirement that either $\Omega^{\prime}$ is bounded or $\mathbb{R}^{n} \backslash \overline{\Omega^{\prime}}$ has a bounded component.

\subsection{Necessity of the constant functions when $n=2$.}

3.2.1. A counterexample. A plausible question to ask, in the case $n=2$, is whether the constant functions can be approximated by fundamental solutions of the Laplacian with singularities on a given pseudo-boundary. Unfortunately, the answer can be negative as happens in the case in which $\Omega^{\prime}$ is the unit disk $D_{1}$ and

$$
\varphi(\mathbf{0}, \boldsymbol{y})=-\frac{1}{2 \pi} \log |\boldsymbol{y}|=0 \quad \text { for every } \quad \boldsymbol{y} \in \partial D_{1} .
$$

Thus every $v \in \mathcal{X}$, where $\mathcal{X}$ is the set of all linear combinations of fundamental solutions with singularities lying on the unit circle, vanishes at the origin, and therefore $\overline{\mathcal{X}} \varsubsetneqq \mathcal{Y}_{\ell}$. On the other hand, in the case of $D_{\varrho}$, the disk of radius $\varrho \neq 1$, the constant function can be approximated by fundamental solutions. In fact, the sequence of MFS solutions

$$
u_{N}(\boldsymbol{x})=\frac{1}{N \log \varrho} \sum_{j=1}^{N} \log \left|\boldsymbol{x}-\varrho \mathrm{e}^{2 \pi \mathrm{i} j / N}\right|, \quad N \in \mathbb{N},
$$

converges to $u \equiv 1$, uniformly in the compact subsets of $D_{\varrho}$. (See [50].)

It is noteworthy that, if the unbounded connected component $U$ of $\mathbb{R}^{n} \backslash \bar{\Omega}$ contains a bounded connected component $U^{\prime}$ of $\mathbb{R}^{n} \backslash \bar{\Omega}^{\prime}$, then the function $\vartheta$ in the proof of Theorem 1 vanishes on $\partial U^{\prime}$, and thus in $\bar{U}^{\prime}$, and consequently in $\mathbb{R}^{n} \backslash \bar{\Omega}$. Therefore, constant functions are not necessary in such case.

3.2.2. A case where the constant functions are not necessary. The constant functions are approximated by linear combinations of fundamental solutions if the pseudo-boundary is included in a unit disk. We have the following result:

Proposition 2. Let $\Omega, \Omega^{\prime}$ be open and bounded domains in $\mathbb{R}^{2}$ with $\Omega$ satisfying the segment condition and $\Omega^{\prime}$ embracing $\Omega$. If $\bar{\Omega}^{\prime}$ is a subset of a unit disk, then the function $f=1_{\bar{\Omega}}$ can be approximated, in the sense of the supremum norm, by linear combinations of the form $\sum_{j=1}^{N} c_{j} \log \left|\boldsymbol{x}-\boldsymbol{y}_{j}\right|$, where $\left\{\boldsymbol{y}_{j}\right\}_{j=1}^{N} \subset \partial \Omega^{\prime}$.

Proof. We may assume that $\bar{\Omega}^{\prime}$ is a subset of the open unit disk $D_{1}$, which is centered at the origin. Let $\mathcal{X}$ be the space of linear combinations of the form $\sum_{j=1}^{N} c_{j} \log \left|\boldsymbol{x}-\boldsymbol{y}_{j}\right|$, where $\left\{\boldsymbol{y}_{j}\right\}_{j=1}^{N} \subset \partial \Omega^{\prime}$. Clearly, the elements of $\mathcal{X}$, when restricted to $\bar{\Omega}$, are also elements of $C(\bar{\Omega})$. Assume that $1_{\bar{\Omega}} \notin \overline{\mathcal{X}}$. Then, due to the Hahn-Banach theorem, there is a measure $\mu \in \mathfrak{M}(\bar{\Omega})=(C(\bar{\Omega}))^{\prime}$, satisfying

$$
\int_{\bar{\Omega}} 1_{\bar{\Omega}} d \mu(\boldsymbol{y})=\mu(\bar{\Omega})=1
$$


and annihilating $\mathcal{X}$. This, as in the proof of Theorem 1 implies that the function

$$
u(\boldsymbol{x})=\int_{\bar{\Omega}} \log |\boldsymbol{x}-\boldsymbol{y}| d \mu(\boldsymbol{y}),
$$

which is harmonic in $\mathbb{R}^{2} \backslash \bar{\Omega}$, vanishes on $\partial \Omega^{\prime}$. Also, for $|\boldsymbol{x}|$ large we have

$$
u(\boldsymbol{x})=\int_{\bar{\Omega}} \log |\boldsymbol{x}| d \mu(\boldsymbol{y})+\int_{\bar{\Omega}} \log \frac{|\boldsymbol{x}-\boldsymbol{y}|}{|\boldsymbol{x}|} d \mu(\boldsymbol{y})=\log |\boldsymbol{x}|+\mathcal{O}\left(\frac{1}{|\boldsymbol{x}|}\right),
$$

which implies that $\lim _{|\boldsymbol{x}| \rightarrow \infty} u(\boldsymbol{x})=+\infty$. Due to the maximum principle we have that $u(\boldsymbol{x})>0$, for every $\boldsymbol{x} \in \mathbb{R}^{2} \backslash \bar{\Omega}^{\prime}$. If we let (identifying $\mathbb{R}^{2}$ with $\mathbb{C}$ )

$$
\tilde{u}(\boldsymbol{x})=\frac{1}{2 \pi} \int_{0}^{2 \pi} \int_{\bar{\Omega}} \log \left|\boldsymbol{x}-\mathrm{e}^{\mathrm{i} \vartheta} \boldsymbol{y}\right| d \mu(\boldsymbol{y}) d \vartheta
$$

then $\tilde{u}$ is harmonic in $\mathbb{R}^{2} \backslash \widetilde{\Omega}$, where $\widetilde{\Omega}=\bigcup_{\vartheta \in[0,2 \pi]} \mathrm{e}^{i \vartheta} \Omega^{\prime}$. In particular, $\widetilde{\Omega}$ is an open disk of radius $\varrho<1$ centered at the origin, where $1-\varrho=\operatorname{dist}\left(\bar{\Omega}^{\prime}, \partial D_{1}\right)$. Also, $\tilde{u}$ is radial, i.e., $\tilde{u}(\boldsymbol{x})=v(|\boldsymbol{x}|)$. In fact, $\tilde{u}$ has to be of the form

$$
\tilde{u}(\boldsymbol{x})=A \log |\boldsymbol{x}|+B,
$$

where $A, B$ are real constants with $A>0$. Clearly, $\tilde{u}(\boldsymbol{x})>0$, for every $|\boldsymbol{x}|=1$, since the same holds for $u$. Thus $B>0$. If we let $R=|\boldsymbol{x}|>|\boldsymbol{y}|=r$, then we have (23, p. 585])

$\frac{1}{2 \pi} \int_{0}^{2 \pi} \log \left|\boldsymbol{x}-\mathrm{e}^{\mathrm{i} \vartheta} \boldsymbol{y}\right| d \vartheta=\frac{1}{2 \pi} \int_{0}^{2 \pi} \frac{1}{2} \log \left(R^{2}-2 r R \cos \vartheta+r^{2}\right) d \vartheta=\log R=\log |\boldsymbol{x}|$.

Using Fubini's theorem we obtain

$$
\tilde{u}(\boldsymbol{x})=\frac{1}{2 \pi} \int_{\bar{\Omega}} \int_{0}^{2 \pi} \log \left|\boldsymbol{x}-\mathrm{e}^{\mathrm{i} \vartheta} \boldsymbol{y}\right| d \vartheta d \mu(\boldsymbol{y})=\mu(\bar{\Omega}) \log |\boldsymbol{x}|=\log |\boldsymbol{x}|,
$$

which contradicts (3.7), and in particular the fact that $B>0$.

\subsubsection{A rescaling argument. The functions}

$$
e_{1}^{R}(\boldsymbol{x})=-\frac{1}{2 \pi} \log \left(\frac{|\boldsymbol{x}|}{R}\right),
$$

are also fundamental solutions of the Laplacian for any $R>0$. Proposition 2 provides that if the pseudo-boundary is a subset of a disk of radius $R$, then the harmonic functions in any domain $\Omega$ embraced by $\Omega^{\prime}$, with boundary satisfying the segment condition, can be approximated by linear combinations of the form $v(\boldsymbol{x})=$ $\sum_{j=1}^{N} c_{j} e_{1}^{R}\left(\boldsymbol{x}-\boldsymbol{y}_{j}\right)$, where $\left\{\boldsymbol{y}_{j}\right\}_{j=1}^{N} \subset \partial \Omega^{\prime}$. This observation allows us to avoid using constant functions in the MFS approximation by rescaling the fundamental solution.

3.3. Analytic pseudo-boundaries. In most of the numerical implementations of the MFS, the pseudo-boundary is analytic (i.e., circle, ellipse, sphere, torus) or union of analytic surfaces (i.e., polygon, polyhedron, cylinder). In such cases, linear combinations of fundamental solutions with sources on a small part of the pseudoboundary are dense in the set of solutions of the equation under consideration. 
Two-dimensional case. Suppose that the pseudo-boundary $\partial \Omega^{\prime}$ of a bounded domain $\Omega \subset \mathbb{R}^{2}$ (satisfying the segment condition) can be parametrized as

$$
\partial \Omega^{\prime}=\{(\alpha(s), \beta(s)): s \in I\},
$$

where $I$ is an open interval and $\alpha, \beta: I \rightarrow \mathbb{R}$ real analytic functions. Let $\Sigma \subset \partial \Omega^{\prime}$, which has at least one limit point. Then the set

$$
\mathcal{X}=\left\{\sum_{j=1}^{N} c_{j} e_{1}\left(\boldsymbol{x}-\boldsymbol{y}_{j}\right): \boldsymbol{y}_{j} \in \Sigma \text { and } c_{j} \in \mathbb{R}\right\} \oplus\left\{\left.c \cdot 1\right|_{\bar{\Omega}}: c \in \mathbb{R}\right\}
$$

is dense in

$$
\mathcal{Y}_{\ell}=\left\{u \in C^{2}(\Omega): \Delta u=0 \text { in } \Omega\right\} \cap C^{\ell}(\bar{\Omega}),
$$

with respect to the norm of $C^{\ell}(\bar{\Omega})$, where $e_{1}$ is given by (1.6).

In order to prove this, we have to repeat the proof of Theorem 1 In this case, $\vartheta(\boldsymbol{x})=\nu\left(\tau_{\boldsymbol{x}} e_{1}\right)$, vanishes only on $\Sigma$. However, the function

$$
\gamma(s)=\vartheta(\alpha(s), \beta(s)), \quad s \in I,
$$

is real analytic, as a composition of real analytic functions, and since the zero set of $\gamma$ has a limit point in $I$, then $\gamma$ vanishes everywhere in $I$, and consequently, $\vartheta$ vanishes everywhere on $\partial \Omega^{\prime}$.

The higher dimensional case. Let $\Omega, \Omega^{\prime} \subset \mathbb{R}^{n}, n>2$, and $\partial \Omega^{\prime}$ be parametrized as

$$
\partial \Omega^{\prime}=\left\{\left(\alpha_{1}\left(s_{1}, \ldots, s_{n-1}\right), \ldots, \alpha_{n}\left(s_{1}, \ldots, s_{n-1}\right)\right):\left(s_{1}, \ldots, s_{n-1}\right) \in V\right\},
$$

where $\alpha_{1}, \ldots, \alpha_{n}$ are real analytic functions on an open set $V \subset \mathbb{R}^{n-1}$. If $\Sigma$ is an open subset of $\partial \Omega^{\prime}$, which intersects every connected component of $\partial \Omega^{\prime}$, then $\mathcal{X}$, defined by (3.9), is dense in $\mathcal{Y}_{\ell}$, defined by (3.10), with respect to the norm of $C^{\ell}(\bar{\Omega})$. In order to prove this we use the fact that real analytic functions in $V$ which vanish in an open set, which intersects every component of $U$, have to vanish everywhere in $V$. For further discussion and numerical implementations see Alves 7].

\section{BiHARMONIC AND $m$-HARMONIC PROBLEMS}

A function $u$ is called biharmonic if $\Delta^{2} u=0$. More generally, $u$ is called $m$-harmonic (or polyharmonic) if $\Delta^{m} u=0$. In this section, we shall investigate density questions in the case of approximations of solutions of biharmonic and $m$-harmonic operators, i.e., $\mathcal{L}=(-\Delta)^{m}$, by linear combinations of their fundamental solutions.

4.1. Biharmonic problems. The function

$$
e_{2}(\boldsymbol{x})=\left\{\begin{array}{lcc}
\frac{1}{8 \pi}|\boldsymbol{x}|^{2}(\log |\boldsymbol{x}|-1) & \text { if } & n=2, \\
\frac{1}{4 \omega_{3}} \log |\boldsymbol{x}| & \text { if } & n=4, \\
\frac{|\boldsymbol{x}|^{4-n}}{2(4-n)(2-n) \omega_{n-1}} & \text { if } & n \neq 2,4,
\end{array}\right.
$$


is a fundamental solution of the biharmonic operator $\mathcal{L}=\Delta^{2}$. In fact, if $e_{1}$ is the fundamental solution of $-\Delta$ given by (1.6), then $-\Delta e_{2}=e_{1}$, in the sense of distributions. (See [8].)

Browder's Theorem (see footnote 2) guarantees that the linear combinations of the fundamental solutions of the biharmonic operator with singularities in a neighborhood of the pseudo-boundary are dense in the solution space. However, the example that follows shows that the linear combinations of the fundamental solutions of the biharmonic operator with singularities on the pseudo-boundary are not dense in the solution space.

4.1.1. A nondensity result. In general, a biharmonic function $u \in C^{4}(\Omega) \cap C(\bar{\Omega})$, where $\Omega$ is an open bounded domain, cannot be approximated by linear combinations of the fundamental solutions $e_{2}$ in (4.1) with singularities located on a given pseudo-boundary $\partial \Omega^{\prime}$ embracing $\Omega$. Let, for example, $\Omega$ be $B(0, \varrho)$, the threedimensional ball of radius $\varrho$ centered at the origin and let $\partial \Omega^{\prime}$ be $S(0, R)$, the sphere of radius $R$, where $\varrho<R$, also centered at the origin. We shall show that the constant function in $\bar{B}(0, \varrho)$, which is biharmonic, cannot be approximated by linear combinations of the form

$$
u_{N}(\boldsymbol{x})=\sum_{j=1}^{N} c_{j}\left|\boldsymbol{x}-\boldsymbol{y}_{j}\right| \quad \text { where }\left\{\boldsymbol{y}_{j}\right\}_{j=1}^{N} \subset S(0, R) .
$$

If this is not the case, then for every $\varepsilon>0$, there exist $\left\{\boldsymbol{y}_{j}\right\}_{j=1}^{N} \subset S(0, R)$, such that

$$
1-\varepsilon<\sum_{j=1}^{N} c_{j}\left|\boldsymbol{x}-\boldsymbol{y}_{j}\right|<1+\varepsilon,
$$

for every $\boldsymbol{x} \in \bar{B}(0, \varrho)$. If $U$ is a unitary matrix in $\mathbb{R}^{3 \times 3}$, i.e., $U^{T} U=I$, then clearly,

$$
1-\varepsilon<\sum_{j=1}^{N} c_{j}\left|U^{T} \boldsymbol{x}-\boldsymbol{y}_{j}\right|=\sum_{j=1}^{N} c_{j}\left|\boldsymbol{x}-U \boldsymbol{y}_{j}\right|<1+\varepsilon,
$$

for every $\boldsymbol{x} \in \bar{B}(0, \varrho)$ as well. The set $S O(3)$ of unitary matrices in $\mathbb{R}^{3 \times 3}$ is a compact group, with respect to the matrix multiplication. Therefore, $S O(3)$ possesses a Haar measure; an invariant, with respect to multiplication, positive measure $\mu$, which we normalize to be a probability measure. (See [19].) Integrating (4.2) over $S O(3)$ we obtain

$$
1-\varepsilon<\sum_{j=1}^{N} c_{j} \int_{S O(3)}\left|\boldsymbol{x}-U \boldsymbol{y}_{j}\right| d \mu(U)<1+\varepsilon,
$$

for every $\boldsymbol{x} \in \bar{B}(0, \varrho)$. Moreover, the integral $\int_{S O(3)}\left|\boldsymbol{x}-U \boldsymbol{y}_{j}\right| d \mu(U)$ does not depend on $\boldsymbol{y}_{j}$, due to the invariance of $\mu$ and the fact that, for every $\boldsymbol{y} \in S(0, R)$, there exists a $V \in S O(3)$, such that $V \boldsymbol{y}_{j}=\boldsymbol{y}$. Therefore, (4.3) yields that

$$
1-\varepsilon<s_{N} \int_{S O(3)}|\boldsymbol{x}-U \boldsymbol{y}| d \mu(U)<1+\varepsilon
$$


for every $\boldsymbol{x} \in \bar{B}(0, \varrho)$ and $\boldsymbol{y} \in S(0, R)$, where $s_{N}=\sum_{j=1}^{N} c_{j}$. Subsequently setting $f(\boldsymbol{x})=\int_{S O(3)}|\boldsymbol{x}-U \boldsymbol{y}| d \mu(U)$ and using Fubini's theorem we obtain

$$
\begin{aligned}
f(\boldsymbol{x}) & =\frac{1}{4 \pi R^{2}} \int_{S(0, R)}\left(\int_{S O(3)}|\boldsymbol{x}-U \boldsymbol{y}| d \mu(U)\right) d \boldsymbol{y} \\
& =\frac{1}{4 \pi R^{2}} \int_{S O(3)}\left(\int_{S(0, R)}|\boldsymbol{x}-U \boldsymbol{y}| d \boldsymbol{y}\right) d \mu(U)=\frac{1}{4 \pi R^{2}} \int_{S(0, R)}|\boldsymbol{x}-\boldsymbol{y}| d \boldsymbol{y},
\end{aligned}
$$

since $\int_{S(0, R)}|\boldsymbol{x}-U \boldsymbol{y}| d \boldsymbol{y}$ does not depend on $\boldsymbol{y} \in S(0, R)$ nor on $U \in S O(3)$. Clearly, $f$ is a function of $r=|\boldsymbol{x}|$. In particular, if we set $\boldsymbol{x}=r(1,0,0)$ and $\boldsymbol{y}=\left(s, y_{1}, y_{2}\right) \in S(0, R)$, then $|\boldsymbol{x}-\boldsymbol{y}|=\left(R^{2}-2 r s+r^{2}\right)^{1 / 2}$ and integration over the sphere $S(0, R)$ reduces to

$$
\begin{aligned}
f(\boldsymbol{x}) & =\frac{1}{4 \pi R^{2}} \int_{-R}^{R} 2 \pi R\left(R^{2}-2 r s+r^{2}\right)^{1 / 2} d s=\left.\frac{1}{2 R} \cdot \frac{2}{3} \cdot \frac{1}{2 r} \cdot\left(R^{2}+2 r s+r^{2}\right)^{3 / 2}\right|_{s=-R} ^{s=R} \\
& =\frac{1}{6 r R}\left(\left(R^{2}+2 r R+r^{2}\right)^{3 / 2}-\left(R^{2}-2 r R+r^{2}\right)^{3 / 2}\right)=\frac{1}{6 r R}\left((R+r)^{3}-(R-r)^{3}\right) \\
& =\frac{1}{6 r R}\left(6 r R^{2}+2 r^{3}\right)=R+\frac{r^{2}}{3 R}=R+\frac{|\boldsymbol{x}|^{2}}{3 R},
\end{aligned}
$$

which contradicts (4.4).

4.1.2. The standard implementation of the MFS. In the MFS for biharmonic problems, the approximate solution is a linear combination of two types of fundamental solutions, the fundamental solutions of the Laplacian as well as the fundamental solutions of the biharmonic operator, i.e.,

$$
u_{N}(\boldsymbol{x} ; \boldsymbol{c}, \boldsymbol{d})=\sum_{j=1}^{N}\left\{c_{j} e_{1}\left(\boldsymbol{x}-\boldsymbol{y}_{j}\right)+d_{j} e_{2}\left(\boldsymbol{x}-\boldsymbol{y}_{j}\right)\right\}, \quad \boldsymbol{x} \in \bar{\Omega},
$$

where the singularities $\boldsymbol{y}_{j}, j=1, \ldots, N$, lie outside of $\bar{\Omega}$. (See [9, 16, 18, 25, 32, 37].) We have the following density result:

Theorem 2. Let $\Omega, \Omega^{\prime}$ be open bounded domains in $\mathbb{R}^{n}, n \geq 3$, with boundary of $\Omega$ satisfying the segment condition and $\Omega^{\prime}$ embracing $\Omega$, and let $\ell$ be a nonnegative integer. Then the space $\mathcal{X}$ of all finite linear combinations of the form

$$
v(\boldsymbol{x})=\sum_{j=1}^{N}\left\{c_{j} e_{1}\left(\boldsymbol{x}-\boldsymbol{y}_{j}\right)+d_{j} e_{2}\left(\boldsymbol{x}-\boldsymbol{y}_{j}\right)\right\},
$$

restricted to $\bar{\Omega}$, where $\left\{\boldsymbol{y}_{j}\right\}_{j=1}^{N} \subset \partial \Omega^{\prime}$, is dense in

$$
\mathcal{Y}_{\ell}=\left\{u \in C^{4}(\Omega): \Delta^{2} u=0 \text { in } \Omega\right\} \cap C^{\ell}(\bar{\Omega})
$$

with respect to the norm of $C^{\ell}(\bar{\Omega})$. If $n=2$, then the same density result holds for the linear sum $\mathcal{X} \oplus\left\{\left.c_{1} \cdot 1\right|_{\bar{\Omega}}+c_{2} \cdot|\boldsymbol{x}|^{2}: c_{1}, c_{2} \in \mathbb{R}\right\}$.

Proof. Let $\nu \in\left(C^{\ell}(\bar{\Omega})\right)^{\prime}$ annihilating all the elements of $\mathcal{X}$, and $\omega_{j}=e_{j} * \nu, j=1,2$. As in the proof of Theorem 1, we obtain

$$
\left.\omega_{1}\right|_{\partial \Omega^{\prime}}=\left.\omega_{2}\right|_{\partial \Omega^{\prime}}=0 .
$$

Also,

$$
-\Delta \omega_{2}=-\Delta\left(e_{2} * \nu\right)=\left(-\Delta e_{2}\right) * \nu=e_{1} * \nu=\omega_{1},
$$


in the sense of distributions, since $-\Delta e_{2}=e_{1}$, also in the sense of distributions. As in the proof of Theorem 11, we obtain $\left.\omega_{1}\right|_{\mathbb{R}^{n} \backslash \bar{\Omega}}=0$. In particular, since $\omega_{1}, \omega_{2}$ are real analytic functions in $\mathbb{R}^{n} \backslash \bar{\Omega}$, we deduce that $\Delta \omega_{2}=0$ in $\mathbb{R}^{n} \backslash \bar{\Omega}$.

If $n>4$, we have

$$
e_{2}(\boldsymbol{x})=\frac{1}{2(4-n)(2-n) \omega_{n-1}}|\boldsymbol{x}|^{4-n} \quad \text { and } \quad D^{\alpha} e_{2}(\boldsymbol{x})=\mathcal{O}\left(|\boldsymbol{x}|^{4-n-|\alpha|}\right),
$$

for every multi-index $\alpha$. Therefore, for $|\boldsymbol{x}|$ large,

$$
\omega_{2}(\boldsymbol{x})=\left(e_{2} * \nu\right)(\boldsymbol{x})=\left\langle\tau_{\boldsymbol{x}} \breve{e}_{2}, \nu\right\rangle=\left\langle\tau_{\boldsymbol{x}} e_{2}, \nu\right\rangle=\mathcal{O}\left(|\boldsymbol{x}|^{4-n}\right) .
$$

Therefore, $\lim _{|\boldsymbol{x}| \rightarrow \infty} \omega_{2}(\boldsymbol{x})=0$ and combining this with (4.8) we conclude that $\left.\omega_{2}\right|_{\mathbb{R}^{n} \backslash \bar{\Omega}}=0$.

If $n=4$, then $e_{2}(\boldsymbol{x})=\frac{\log |\boldsymbol{x}|}{4 \omega_{3}}$ and $D^{\alpha} e_{2}(\boldsymbol{x})=\mathcal{O}\left(|\boldsymbol{x}|^{-|\alpha|}\right)$, for every multiindex $\alpha$. Theorem 1 provides that the function $u=\left.1\right|_{\bar{\Omega}}$ belongs to $\overline{\mathcal{X}}$, the closure of $\mathcal{X}$ with respect to the norm of $C^{\ell}(\bar{\Omega})$. Repeating what was done in (3.3)-(3.4) we obtain, for $|\boldsymbol{x}|$ large,

$$
\omega_{2}(\boldsymbol{x})=\mathcal{O}\left(\frac{1}{|\boldsymbol{x}|}\right)
$$

which also implies that $\lim _{|\boldsymbol{x}| \rightarrow \infty} \omega_{2}(\boldsymbol{x})=0$ and consequently that $\left.\omega_{2}\right|_{\mathbb{R}^{4} \backslash \bar{\Omega}}=0$.

If $n=3$, then $e_{2}(\boldsymbol{x})=-\frac{1}{8 \pi}|\boldsymbol{x}|$, and for $|\boldsymbol{x}|$ large and $\boldsymbol{y} \in \bar{\Omega}$ we have

$$
\begin{aligned}
|\boldsymbol{x}-\boldsymbol{y}|-|\boldsymbol{x}|+\frac{1}{|\boldsymbol{x}|} \boldsymbol{x} \cdot \boldsymbol{y} & =\frac{|\boldsymbol{y}|^{2}\left(|\boldsymbol{x}|^{2}+|\boldsymbol{x}| \cdot|\boldsymbol{x}-\boldsymbol{y}|+2 \boldsymbol{x} \cdot \boldsymbol{y}\right)-4(\boldsymbol{x} \cdot \boldsymbol{y})^{2}}{|\boldsymbol{x}|(|\boldsymbol{x}-\boldsymbol{y}|+|\boldsymbol{x}|)^{2}} \\
& =\mathcal{R}(\boldsymbol{x}, \boldsymbol{y}),
\end{aligned}
$$

and, clearly, for $|\boldsymbol{x}|$ large,

$$
D_{\boldsymbol{y}}^{\alpha} \mathcal{R}(\boldsymbol{x}, \boldsymbol{y})=\mathcal{O}\left(\frac{1}{|\boldsymbol{x}|^{1+|\alpha|}}\right),
$$

for all multi-indices $\alpha$. The functions $\left.1\right|_{\Omega}$ and $p_{i}(\boldsymbol{x})=x_{i}, i=1,2,3$, are harmonic, and thus Theorem 1 implies that $\left.1\right|_{\bar{\Omega}}, p_{1}, p_{2}, p_{3} \in \overline{\mathcal{X}}$. Hence

$$
\nu\left(1_{\bar{\Omega}}\right)=\nu\left(p_{1}\right)=\nu\left(p_{2}\right)=\nu\left(p_{3}\right)=0 .
$$

Therefore, for $|\boldsymbol{x}|$ large, combining (4.9)-(4.11), we obtain

$$
\nu(|\cdot-\boldsymbol{x}|)=\nu\left(|\boldsymbol{x}| \cdot 1_{\bar{\Omega}}\right)-\frac{1}{|\boldsymbol{x}|} \sum_{j=1}^{3} \nu\left(p_{j}\right)+\nu(\mathcal{R}(\boldsymbol{x}, \cdot))=\mathcal{O}\left(\frac{1}{|\boldsymbol{x}|}\right) .
$$

Note that in the above the functions paired with $\nu$ are functions of $\boldsymbol{y}$ while the variable $\boldsymbol{x}$ is fixed. Therefore, $\omega_{2}(\boldsymbol{x})=\nu\left(\tau_{\boldsymbol{x}} e_{2}\right)=-\frac{1}{8 \pi} \nu(|\cdot-\boldsymbol{x}|) \rightarrow 0$ as $|\boldsymbol{x}| \rightarrow \infty$ and consequently $\left.\omega_{2}\right|_{\mathbb{R}^{3} \backslash \bar{\Omega}}=0$.

If $n=2$, then the functions $\left.1\right|_{\bar{\Omega}}, p_{1}, p_{2}, p_{1} p_{2}, p_{1}^{2}-p_{2}^{2}$ are harmonic in $\mathbb{R}^{2}$. Theorem 1 provides that $\left.1\right|_{\bar{\Omega}}, p_{1}, p_{2}, p_{1} p_{2}, p_{1}^{2}-p_{2}^{2} \in \overline{\mathcal{X}}$. We have also assumed that $|\boldsymbol{y}|^{2}=p_{1}^{2}+p_{2}^{2} \in \overline{\mathcal{X}}$, and therefore $p_{1}^{2}, p_{2}^{2} \in \overline{\mathcal{X}}$. Thus for every $\boldsymbol{x} \in \mathbb{R}^{n}$, we have

$$
|\boldsymbol{x}-\boldsymbol{y}|^{2}=\left.|\boldsymbol{x}|^{2} \cdot 1\right|_{\bar{\Omega}}-2 x_{1} \cdot p_{1}-2 x_{2} \cdot p_{2}+p_{1}^{2}+p_{2}^{2} \in \overline{\mathcal{X}} .
$$


Also, for $|\boldsymbol{x}|$ large, the Taylor expansion around $\boldsymbol{x}$ yields

$$
\begin{aligned}
|\boldsymbol{x}-\boldsymbol{y}|^{2} \log |\boldsymbol{x}-\boldsymbol{y}|^{2}= & |\boldsymbol{x}|^{2} \log |\boldsymbol{x}|^{2}-2\left(\log |\boldsymbol{x}|^{2}+1\right)(\boldsymbol{x} \cdot \boldsymbol{y})+\left(\log |\boldsymbol{x}|^{2}+1\right)|\boldsymbol{y}|^{2} \\
& +\frac{6}{|\boldsymbol{x}|^{2}}(\boldsymbol{x} \cdot \boldsymbol{y})^{2}+\mathcal{R}(\boldsymbol{x}, \boldsymbol{y}),
\end{aligned}
$$

with

$$
D_{\boldsymbol{y}}^{\alpha} \mathcal{R}(\boldsymbol{x}, \boldsymbol{y})=\mathcal{O}\left(|\boldsymbol{x}|^{-1-|\alpha|}\right),
$$

for all multi-indices $\alpha$. Clearly, $|\boldsymbol{x}-\boldsymbol{y}|^{2} \log |\boldsymbol{x}-\boldsymbol{y}|^{2}-\mathcal{R}(\boldsymbol{x}, \boldsymbol{y}) \in \overline{\mathcal{X}}$. Thus

$$
\begin{aligned}
\omega_{2}(\boldsymbol{x}) & =\nu\left(\tau_{\boldsymbol{x}} e_{2}\right)=\frac{1}{8 \pi} \nu\left(|\cdot-\boldsymbol{x}|^{2} \log |\cdot-\boldsymbol{x}|\right)-\frac{1}{8 \pi} \nu\left(|\cdot-\boldsymbol{x}|^{2}\right)=\nu(\mathcal{R}(\boldsymbol{x}, \cdot)) \\
& =\mathcal{O}\left(\frac{1}{|\boldsymbol{x}|}\right) .
\end{aligned}
$$

Thus $\lim _{|\boldsymbol{x}| \rightarrow \infty} \omega_{2}(\boldsymbol{x})=0$ and consequently $\left.\omega_{2}\right|_{\mathbb{R}^{2} \backslash \bar{\Omega}}=0$.

Lemma 1 (with $\mathcal{L}=\Delta^{2}$ and $e=e_{2}$ ) implies that there exists a sequence $\left\{\vartheta^{k}\right\}_{k \in \mathbb{N}} \subset \mathscr{D}^{\prime}(\Omega)$, such that $\operatorname{supp} \vartheta^{k} \subset \Omega,\left\{\Delta^{2} \vartheta^{k}\right\}_{k \in \mathbb{N}} \subset\left(C^{\ell}(\bar{\Omega})\right)^{\prime}$ and $\Delta^{2} \vartheta^{k} \rightarrow \nu$ in the weak ${ }^{\star}$ sense of $C^{\ell}(\bar{\Omega})$. Let $u \in \mathcal{Y}_{\ell}$. Since $\operatorname{supp} \vartheta^{k} \subset \Omega$, then there exists a $\varphi \in \mathscr{D}(\Omega)$, which is equal to 1 in a neighborhood of $\operatorname{supp} \vartheta^{k}$. Clearly, $\Delta^{2} \vartheta^{k}(u)=$ $\Delta^{2} \vartheta^{k}(\varphi u)$, since $u$ and $\varphi u$ coincide in a neighborhood of $\operatorname{supp} \vartheta^{k}$. On the other hand, $\varphi u \in \mathscr{D}(\Omega)$, since $u$ is real analytic in $\Omega$, and thus

$$
\Delta^{2} \vartheta^{k}(u)=\Delta^{2} \vartheta^{k}(\varphi u)=\left\langle\varphi u, \Delta^{2} \vartheta^{k}\right\rangle=\left\langle\Delta^{2}(\varphi u), \vartheta^{k}\right\rangle=0,
$$

since $\Delta^{2}(\varphi u)$ vanishes in a neighborhood of $\operatorname{supp} \vartheta^{k}$. Note that $\varphi u$ is an element of $C^{\ell}(\bar{\Omega})$ and $\mathscr{D}(\Omega)$ while $\Delta^{2} \vartheta^{k}$ is an element of $\left(C^{\ell}(\bar{\Omega})\right)^{\prime}$ and $\mathscr{D}^{\prime}(\Omega)$ and the pairings $\Delta^{2} \vartheta^{k}(\varphi u)$ and $\left\langle\varphi u, \Delta^{2} \vartheta^{k}\right\rangle$ coincide. Finally, we have

$$
\nu(u)=\lim _{k \rightarrow \infty} \Delta^{2} \vartheta^{k}(u)=0,
$$

which concludes the proof.

4.2. $\boldsymbol{m}$-harmonic problems. It can be readily shown that the function

$$
e_{m}(\boldsymbol{x})= \begin{cases}\frac{(-1)^{m}|\boldsymbol{x}|^{2 m-2}\left(\log |\boldsymbol{x}|-\gamma_{m-1}\right)}{2^{2 m-1} \pi((m-1) !)^{2}}, & \text { if } n=2, \\ \frac{(-1)^{m-1}|\boldsymbol{x}|^{2 m-3}}{4 \pi(2 m-3) !}, & \text { if } n=3,\end{cases}
$$

where $\gamma_{0}=0$ and $\gamma_{m}=1+\frac{1}{2}+\cdots+\frac{1}{m}$, for $m \geq 1$, is a fundamental solution of the $m$-harmonic operator $\mathcal{L}=(-\Delta)^{m}$. In fact, these fundamental solutions satisfy the equations

$$
(-\Delta)^{j} e_{k}=e_{k-j}, \quad \text { for } \quad k>j
$$

in the sense of distributions. For $n>3$, the functions

$$
e_{m}(\boldsymbol{x})=|\boldsymbol{x}|^{2 m-n}\left(a_{m, n} \log |\boldsymbol{x}|+b_{m, n}\right),
$$

are fundamental solutions of $(-\Delta)^{m}$, for suitable coefficients $a_{m, n}, b_{m, n}$. In particular, there are unique $a_{m, n}$ and $b_{m, n}$, so that (1.2) and (4.12) are satisfied. In fact, $a_{m, n}=0$ if $2 m<n$ or if $n$ is odd, whereas $b_{m, n}=0$ if $m \geq 0$ and $n$ is even ([8]). 
In the MFS the solutions of $(-\Delta)^{m} u=0$ are approximated by linear combinations of the form

$$
v(\boldsymbol{x})=\sum_{k=1}^{m} \sum_{j=1}^{N} c_{j}^{k} e_{k}\left(\boldsymbol{x}-\boldsymbol{y}_{j}\right),
$$

where the $\boldsymbol{y}_{j}$ 's lie on prescribed pseudo-boundary. The following theorem is a predictable generalization of Theorems 1 and 2 .

Theorem 3. Let $\Omega, \Omega^{\prime}$ be open bounded domains in $\mathbb{R}^{n}, n \geq 3$, with the boundary of $\Omega$ satisfying the segment condition and $\Omega^{\prime}$ embracing $\Omega$, and let $\ell$ be a nonnegative integer. Then the space $\mathcal{X}$ of all finite linear combinations of the form (4.14), when restricted in $\bar{\Omega}$, where $\left\{\boldsymbol{y}_{j}\right\}_{j=1}^{N} \subset \partial \Omega^{\prime}$, is dense in

$$
\mathcal{Y}_{\ell}=\left\{u \in C^{2 m}(\Omega):(-\Delta)^{m} u=0 \text { in } \Omega\right\} \cap C^{\ell}(\bar{\Omega}),
$$

with respect to the norm of $C^{\ell}(\bar{\Omega})$.

If $n=2$, then the same density result holds for the sum

$$
\mathcal{X} \oplus\left\{\left.c_{1} \cdot 1\right|_{\Omega}+c_{2} \cdot|\boldsymbol{x}|^{2}+\cdots+c_{m} \cdot|\boldsymbol{x}|^{2 m-2}: c_{1}, c_{2}, \ldots, c_{m} \in \mathbb{R}\right\} .
$$

Remark 4.1. It can be readily seen that, in the two space dimensions, Proposition 2 allows us to avoid the use of the functions $1,|\boldsymbol{x}|^{2}, \ldots,|\boldsymbol{x}|^{2 m-2}$ in the MFS approximation provided that the fundamental solution of the Laplacian is suitably rescaled. In particular, if $\bar{\Omega}^{\prime} \subset D_{R}$, where $D_{R}$ is a disk of radius $R$, then linear combinations of the form

$$
u_{N}(\boldsymbol{x})=\sum_{j=1}^{N} c_{j}^{1} \varphi_{R}\left(\boldsymbol{x}-\boldsymbol{y}_{j}\right)+\sum_{k=2}^{m} \sum_{j=1}^{N} c_{j}^{k} e_{k}\left(\boldsymbol{x}-\boldsymbol{y}_{j}\right),
$$

where $e_{1}^{R}$ is given by (3.8) and $\left\{\boldsymbol{y}_{j}\right\} \subset \partial \Omega^{\prime}$, are dense in

$$
\mathcal{Y}_{\ell}=\left\{u \in C^{2 m}(\Omega):(-\Delta)^{m} u=0 \text { in } \Omega\right\} \cap C^{\ell}(\bar{\Omega}),
$$

with respect to the norm of $C^{\ell}(\bar{\Omega})$.

4.3. Using Almansi's representation. Almansi [5] showed that every biharmonic function $w$ in $B_{r}\left(\boldsymbol{x}_{0}\right)$, the ball of radius $r$ and center $\boldsymbol{x}_{0}$, can be represented as

$$
w(\boldsymbol{x})=u_{1}(\boldsymbol{x})+\left|\boldsymbol{x}-\boldsymbol{x}_{0}\right|^{2} u_{2}(\boldsymbol{x}),
$$

where $u_{1}$ and $u_{2}$ are harmonic functions in the same ball. In [6], Almansi showed that every $m$-harmonic function $w$ in $B_{r}$ can be represented as

$$
w(\boldsymbol{x})=u_{1}(\boldsymbol{x})+\left|\boldsymbol{x}-\boldsymbol{x}_{0}\right|^{2} u_{2}(\boldsymbol{x})+\cdots+\left|\boldsymbol{x}-\boldsymbol{x}_{0}\right|^{2 m-2} u_{m}(\boldsymbol{x}),
$$

where $u_{1}, \ldots, u_{m}$ are harmonic in $B_{r}$. Nicolescu [41] proved that Almansi's representation holds even when $B_{r}\left(\boldsymbol{x}_{0}\right)$ is replaced by a star-shaped domain with center $\boldsymbol{x}_{0}$. Karageorghis and Fairweather [26] used the fundamental solutions of Laplace's operator and Almansi's representation (4.16) in order to approximate the solutions of the biharmonic equation.

The following theorem justifies the approach of [26].

Theorem 4. Let $\Omega, \Omega^{\prime}$ be open bounded domains in $\mathbb{R}^{n}, n \geq 3$, with the boundary of $\Omega$ satisfying the segment condition and $\Omega^{\prime}$ embracing $\Omega$. We further assume that 
$\Omega$ is a star-shaped domain with center $\boldsymbol{x}_{0}$. Then, for every nonnegative integer $\ell$, the space $\mathcal{X}$ of all finite linear combinations of the form

$$
v(\boldsymbol{x})=\sum_{k=0}^{m-1} \sum_{j=1}^{N} c_{j}^{k}\left|\boldsymbol{x}-\boldsymbol{x}_{0}\right|^{2 k} e_{1}\left(\boldsymbol{x}-\boldsymbol{y}_{j}\right),
$$

restricted in $\bar{\Omega},\left\{\boldsymbol{y}_{j}\right\}_{j=1}^{N} \subset \partial \Omega^{\prime}$, where $e_{1}$ is given by (1.6), is dense in

$$
\mathcal{Y}_{\ell}=\left\{u \in C^{2 m}(\Omega): \Delta^{m} u=0 \text { in } \Omega\right\} \cap C^{\ell}(\bar{\Omega})
$$

with respect to the norm of $C^{\ell}(\bar{\Omega})$. If $n=2$, then the same density result holds for the sum

$$
\mathcal{X} \oplus\left\{\left.c_{1} \cdot 1\right|_{\Omega}+c_{2} \cdot|\boldsymbol{x}|^{2}+\cdots+c_{m} \cdot|\boldsymbol{x}|^{2 m-2}: c_{1}, c_{2}, \ldots, c_{m} \in \mathbb{R}\right\} .
$$

Proof. Let $u \in \mathcal{Y}_{\ell}$ and $v^{\varepsilon}$ be a linear combination of the form (4.14) such that $\left|v^{\varepsilon}-u\right|_{\ell}<\varepsilon$ (such an $m$-harmonic function $v^{\varepsilon}$ exists as a consequence of Theorem 3). Also, let $\Omega^{\prime \prime}$ be a star-shaped open domain with center $\boldsymbol{x}_{0}$ such that

$$
\bar{\Omega} \subset \Omega^{\prime \prime} \subset \bar{\Omega}^{\prime \prime} \subset \Omega^{\prime} .
$$

One way to define such a domain $\Omega^{\prime \prime}$ is as follows. Let $2 d=\operatorname{dist}\left(\partial \Omega, \mathbb{R}^{n} \backslash \Omega^{\prime}\right)>0$ and define

$$
\Omega^{\prime \prime}=\left\{\boldsymbol{x} \in \mathbb{R}^{n}: \exists \boldsymbol{y} \in \partial \Omega \text { such that } \boldsymbol{x}-\boldsymbol{x}_{0}=\alpha\left(\boldsymbol{y}-\boldsymbol{x}_{0}\right) \text { for some } \alpha \in(0,1+d)\right\} .
$$

The $m$-harmonic function $v^{\varepsilon}$ can be expressed as

$$
v^{\varepsilon}(\boldsymbol{x})=u_{1}^{\varepsilon}(\boldsymbol{x})+\left|\boldsymbol{x}-\boldsymbol{x}_{0}\right|^{2} u_{2}^{\varepsilon}(\boldsymbol{x})+\cdots+\left|\boldsymbol{x}-\boldsymbol{x}_{0}\right|^{2 m-2} u_{m}^{\varepsilon}(\boldsymbol{x}), \quad \boldsymbol{x} \in \Omega^{\prime \prime},
$$

where $u_{1}^{\varepsilon}, \ldots, u_{m}^{\varepsilon}$ are harmonic in $\Omega^{\prime \prime}$.

Theorem 1 provides that each of the $u_{k}^{\varepsilon}$ 's, when restricted in $\bar{\Omega}$, can be approximated, with respect to the $|\cdot|_{\ell}-$ norm, by linear combinations of the form

$$
u_{k}^{\varepsilon, \delta}(\boldsymbol{x})=\sum_{j=1}^{N_{\varepsilon, \delta}} c_{k, j} e_{1}\left(\boldsymbol{x}-\boldsymbol{y}_{j}\right), \quad \text { where } \quad\left\{\boldsymbol{y}_{j}\right\}_{j=1}^{N_{\varepsilon, \delta}} \subset \partial \Omega^{\prime} .
$$

In particular, these $u_{k}^{\varepsilon, \delta}$, s can be chosen so that $\left|v^{\varepsilon}-v^{\varepsilon, \delta}\right|_{\ell}<\delta$, where

$$
v^{\varepsilon, \delta}(\boldsymbol{x})=u_{1}^{\varepsilon, \delta}(\boldsymbol{x})+\left|\boldsymbol{x}-\boldsymbol{x}_{0}\right|^{2} u_{2}^{\varepsilon, \delta}(\boldsymbol{x})+\cdots+\left|\boldsymbol{x}-\boldsymbol{x}_{0}\right|^{2 m-2} u_{m}^{\varepsilon, \delta}(\boldsymbol{x}),
$$

which concludes the proof.

\section{Approximations By Fundamental SOlutions of the Modified HELMHOLTZ OPERATOR}

5.1. The modified Helmholtz equation. The elliptic partial differential operator $\mathcal{L}=\Delta-\kappa^{2}$, for $\kappa>0$, corresponds to the modified Helmholtz equation:

$$
\mathcal{L} u=\left(\Delta-\kappa^{2}\right) u=0,
$$

and has as a fundamental solution the function

$$
e_{1}\left(\boldsymbol{x}, \kappa^{2}\right)=\left\{\begin{array}{llc}
-\frac{K_{0}(\kappa|\boldsymbol{x}|)}{2 \pi} & \text { if } & n=2, \\
-\frac{\mathrm{e}^{-\kappa|\boldsymbol{x}|}}{4 \pi|\boldsymbol{x}|} & \text { if } & n=3,
\end{array}\right.
$$


where $K_{0}(r)$ is the modified Bessel function of the second kind. In fact, the function $e_{1}\left(\boldsymbol{x}, \kappa^{2}\right)$ given by (5.2) is a fundamental solution for $\mathcal{L}=\Delta-\kappa^{2}$, even in the case $\kappa \in \mathbb{C}$ for $n=3$ and $\kappa \in \mathbb{C} \backslash\{0\}$ for $n=2$.

We have the following density result:

Theorem 5. Let $\Omega, \Omega^{\prime}$ be open bounded domains in $\mathbb{R}^{n}, n=2,3$, with $\Omega$ satisfying the segment condition and $\Omega^{\prime}$ embracing $\Omega$, and let $\ell$ be a nonnegative integer. Then the space $\mathcal{X}$ of linear combinations of the form $\sum_{j=1}^{N} c_{j} e\left(\boldsymbol{x}-\boldsymbol{y}_{j}, \kappa^{2}\right)$, where $N \in \mathbb{N}$, $\kappa$ positive, $e_{1}\left(\cdot, \kappa^{2}\right)$ is given by (5.2) and $\left\{\boldsymbol{y}_{j}\right\}_{j=1}^{N}$ lie on $\partial \Omega^{\prime}$, is dense in

$$
\mathcal{Y}_{\ell}=\left\{u \in C^{2}(\Omega):\left(\Delta-\kappa^{2}\right) u=0 \text { in } \Omega\right\} \cap C^{\ell}(\bar{\Omega})
$$

with respect to the norm of $C^{\ell}(\bar{\Omega})$.

Proof. We only need to show that, for every $\nu \in \mathcal{Y}_{\ell}^{\perp} \subset\left(C^{\ell}(\bar{\Omega})\right)^{\prime}$, the convolution $\vartheta=e_{1}\left(\cdot, \kappa^{2}\right) * \nu$, which satisfies $\left(\Delta-\kappa^{2}\right) \vartheta=0$ in $\mathbb{R}^{n} \backslash \bar{\Omega}$, vanishes in $\mathbb{R}^{n} \backslash \bar{\Omega}$, and the rest of the proof is a tedious repetition of the proof of Theorem 1 .

In order to prove that $\left.\vartheta\right|_{\mathbb{R}^{n} \backslash \bar{\Omega}}=0$, we need the following facts:

(i) $\lim _{|\boldsymbol{x}| \rightarrow \infty} D^{\alpha} e_{1}\left(\boldsymbol{x}, \kappa^{2}\right)=0$, for every multi-index $\alpha$, which in turn implies that $\lim _{|\boldsymbol{x}| \rightarrow \infty} \vartheta(\boldsymbol{x})=0$.

(ii) the maximum principle for the modified Helmholtz equation:

Let $V$ be an open bounded subset of $\mathbb{R}^{n}$. If $u \in C^{2}(V)$ satisfies (5.1) in $V$, then

$$
\sup _{\boldsymbol{x} \in V}|u(\boldsymbol{x})|=\sup _{\boldsymbol{x} \in \partial V}|u(\boldsymbol{x})| .
$$

Fact (i) follows from the definition of $e_{1}\left(\cdot, \kappa^{2}\right)$ and of the fact that the function $K_{0}$ is real analytic in $(0, \infty)$, it blows up at $r=0$ and (see [1, pp. 374-378].)

$$
\frac{d^{j}}{d r^{j}} K_{0}(r) \simeq \sqrt{\frac{\pi}{2 r}} \mathrm{e}^{-r},
$$

for $r$ large and for every nonnegative integer $j$. For a proof of fact (ii) see [20].

5.2. Approximation of solutions of poly-Helmholtz equations. The higher order equation

$$
\left(\Delta-\kappa_{1}^{2}\right) \cdots\left(\Delta-\kappa_{m}^{2}\right) u=0,
$$

which is also elliptic and is known as a poly-Helmholtz equation, arises from $m$-porosity media as well as from $m$-layered aquifer systems; see [12] and the references therein.

5.2.1. Construction of fundamental solutions. Following the technique of Trèves [54] (see also [12]), fundamental solutions of operators of the form

$$
\mathcal{L}=\prod_{j=1}^{m}\left(\Delta-\kappa_{j}^{2}\right),
$$

where $\kappa_{1}, \ldots, \kappa_{m}$ are distinct nonnegative reals, can be constructed as linear combinations of fundamental solutions of the operators $\mathcal{L}_{j}=\Delta-\kappa_{j}^{2}, j=1, \ldots, m$. 
Let $\varphi_{m}(\boldsymbol{x})=\sum_{j=1}^{m} \beta_{j} e_{1}\left(\boldsymbol{x}, \kappa_{j}^{2}\right)$, where $e_{1}\left(\cdot, \kappa^{2}\right)$ is given by (5.2) and $\kappa_{1}^{2}, \ldots, \kappa_{m}^{2}$ distinct. Then

$$
\begin{aligned}
\left(\Delta-\kappa_{1}^{2}\right) \varphi_{m} & =\sum_{j=1}^{m} \beta_{j}\left(\Delta-\kappa_{1}^{2}\right) e_{1}\left(\cdot, \kappa_{j}^{2}\right)=\sum_{j=1}^{m} c_{j}\left(\delta+\left(\kappa_{j}^{2}-\kappa_{1}^{2}\right) e_{1}\left(\cdot, \kappa_{j}^{2}\right)\right) \\
& =\left(\sum_{j=1}^{m} \beta_{j}\right) \delta+\sum_{j=2}^{m} \beta_{j}\left(\kappa_{j}^{2}-\kappa_{1}^{2}\right) e_{1}\left(\cdot, \kappa_{j}^{2}\right) .
\end{aligned}
$$

We obtain the first equation for the $\beta_{j}$ 's setting the coefficient of $\delta$ equal to zero, i.e., $\sum_{j=1}^{m} \beta_{j}=0$. After applying the first $\ell$ factors of the operator $\mathcal{L}$ we get

$$
\prod_{j=1}^{\ell}\left(\Delta-\kappa_{j}^{2}\right) \varphi_{m}=\left(\sum_{j=\ell}^{m} \beta_{j}\left(\prod_{i=1}^{\ell-1}\left(\kappa_{j}^{2}-\kappa_{i}^{2}\right)\right)\right) \delta+\sum_{j=\ell+1}^{m} \beta_{j}\left(\prod_{i=1}^{\ell}\left(\kappa_{j}^{2}-\kappa_{i}^{2}\right)\right) e_{1}\left(\cdot, \kappa_{j}^{2}\right)
$$

and the $\ell^{\text {th }}$-equation is

$$
\sum_{j=\ell}^{m} \beta_{j}\left(\prod_{i=1}^{\ell-1}\left(\kappa_{j}^{2}-\kappa_{i}^{2}\right)\right)=0
$$

Finally, applying all factors of the operator $\mathcal{L}$ we have

$$
\mathcal{L} \varphi_{m}=\beta_{m}\left(\kappa_{m}^{2}-\kappa_{1}^{2}\right) \cdots\left(\kappa_{m}^{2}-\kappa_{m-1}^{2}\right) \delta
$$

and the last equation is $\beta_{m}\left(\kappa_{m}^{2}-\kappa_{1}^{2}\right) \cdots\left(\kappa_{m}^{2}-\kappa_{1}^{2}\right)=1$. We have thus obtained an upper diagonal system, with unknowns $\beta_{1}, \ldots, \beta_{m}$, the solution of which is

$$
\beta_{j}=\left(\prod_{\substack{\ell=1 \\ \ell \neq j}}^{m}\left(\kappa_{j}^{2}-\kappa_{\ell}^{2}\right)\right)^{-1}, \quad j=1, \ldots, m
$$

One can easily show that

$$
\left(\Delta-\kappa_{m}^{2}\right) \varphi_{m}=\varphi_{m-1},
$$

in the sense of distributions, where $\varphi_{m-1}$ is the fundamental solution of the operator $\mathcal{L}=\left(\Delta-\kappa_{1}^{2}\right) \cdots\left(\Delta-\kappa_{m-1}^{2}\right)$, constructed analogously.

If $m=2$, then $e^{\mathcal{L}}(\boldsymbol{x})=\frac{e_{1}\left(\boldsymbol{x}, \lambda^{2}\right)-e_{1}\left(\boldsymbol{x}, \kappa^{2}\right)}{\lambda^{2}-\kappa^{2}}$, is a fundamental solution of $\mathcal{L}=$ $\left(\Delta-\kappa^{2}\right)\left(\Delta-\lambda^{2}\right)$, provided $\kappa^{2} \neq \lambda^{2}$.

Letting $\lambda \rightarrow \kappa$, we obtain a fundamental solution of $\mathcal{L}=\left(\Delta-\kappa^{2}\right)^{2}$, namely,

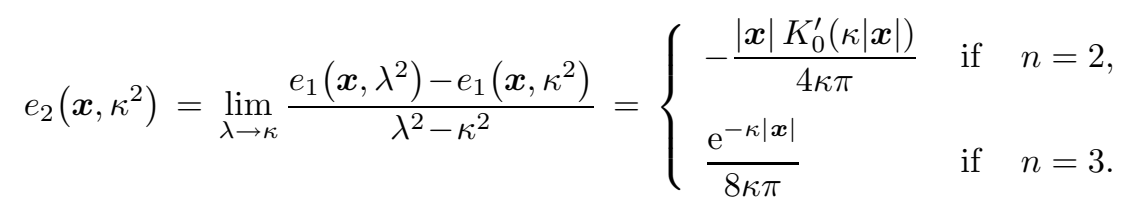

In particular, when $\lambda=0$ and $\kappa \neq 0$, the function

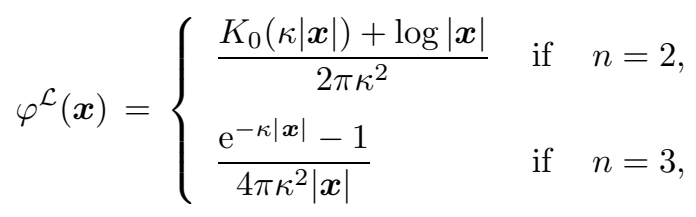


is a fundamental solution of the operator $\mathcal{L}=\Delta^{2}-\kappa^{2} \Delta$. Fundamental solutions of operators of the form

$$
\mathcal{L}=\left(\Delta-\kappa_{1}^{2}\right)^{\nu_{1}} \cdots\left(\Delta-\kappa_{m}^{2}\right)^{\nu_{m}}
$$

can be obtained in a similar fashion. It can be readily shown that the function (see for details [54])

$$
\varphi^{\mathcal{L}}(\boldsymbol{x})=\left.\sum_{j=1}^{m} \frac{1}{\left(\nu_{j}-1\right) !} \frac{\partial^{\nu_{j}-1}\left(\beta_{j} e_{1}(\boldsymbol{x}, \lambda)\right)}{\partial \lambda^{\nu_{j}-1}}\right|_{\lambda=\kappa_{j}^{2}},
$$

is a fundamental solution of the operator in (5.7), where

$$
\beta_{j}=\prod_{\substack{\ell=1 \\ \ell \neq j}}^{m} \frac{1}{\left(\kappa_{j}^{2}-\kappa_{\ell}^{2}\right)^{\nu_{j}}}
$$

In particular, the function

$$
e_{m}\left(\boldsymbol{x}, \kappa^{2}\right)=\left.\frac{\partial^{m-1} e_{1}(\boldsymbol{x}, \lambda)}{\partial \lambda^{m-1}}\right|_{\lambda=\kappa^{2}}
$$

is a fundamental solution of $\left(\Delta-\kappa^{2}\right)^{m}$ and the following equation

$$
\left(\Delta-\kappa^{2}\right)^{j} e_{\ell}\left(\cdot, \kappa^{2}\right)=e_{\ell-j}\left(\cdot, \kappa^{2}\right)
$$

is satisfied in the sense of distributions. Note that $\varphi^{\mathcal{L}}$ in (5.8) is a linear combination of the functions

$$
e_{\ell}\left(\cdot ; \kappa_{j}^{2}\right), \quad j=1, \ldots, m, \ell=1, \ldots, \nu_{j} .
$$

5.2.2. A density result. Following the lines of the proof of Theorem 2, we obtain the following result:

Theorem 6. Let $\Omega, \Omega^{\prime}$ be open bounded domains in $\mathbb{R}^{n}, n=2,3$, with $\Omega$ satisfying the segment condition and $\Omega^{\prime}$ embracing $\Omega$, and let $\ell \in \mathbb{N}$. Further, assume that $0 \leq \kappa_{1}<\cdots<\kappa_{m}$. If $n=3$ or $k_{1}>0$, then the space $\mathcal{X}$ of finite linear combinations of the form

$$
u^{N}(\boldsymbol{x})=\sum_{i=1}^{N} \sum_{j=1}^{m} c_{i j} e_{1}\left(\boldsymbol{x}-\boldsymbol{y}_{i}, \kappa_{j}^{2}\right),
$$

where $e_{1}\left(\cdot, \kappa^{2}\right)$ is given by (5.2) and $\boldsymbol{y}_{i}, i=1, \ldots, N$, lying on $\partial \Omega^{\prime}$, is dense in

$$
\mathcal{Y}_{\ell}=\left\{u \in C^{2 m}(\Omega): \mathcal{L} u=0 \text { in } \Omega\right\} \cap C^{\ell}(\bar{\Omega}),
$$

with respect to the norm of $C^{\ell}(\bar{\Omega})$, where $\mathcal{L}=\prod_{j=1}^{m}\left(\Delta-\kappa_{j}^{2}\right)$. If $n=2$ and $k_{1}=0$, the result still holds, provided that the constant functions are included in $\mathcal{X}$.

Proof. Assume first that $\kappa_{1}>0$ or $n=3$. If $\nu \in\left(C^{\ell}(\bar{\Omega})\right)^{\prime}$ annihilates $\mathcal{X}$, then $\nu\left(\tau_{\boldsymbol{y}} e_{1}\left(\cdot ; \kappa_{j}^{2}\right)\right)=0$, for every $\boldsymbol{y} \in \partial \Omega^{\prime}$ and $j=1, \ldots, m$. As in the proof of Theorem 2] let

$$
\vartheta_{j}(\boldsymbol{x})=\nu\left(\tau_{\boldsymbol{x}} \varphi_{j}\right)=\left(\varphi_{j} * \nu\right)(\boldsymbol{x}), \quad j=1, \ldots, m,
$$

where $\varphi_{j}$ is the fundamental solution of $\mathcal{L}=\left(\Delta-\kappa_{1}^{2}\right) \cdots\left(\Delta-\kappa_{j}^{2}\right)$ constructed so that (5.4) holds. We need to show that $\left.\vartheta_{m}\right|_{\mathbb{R}^{n} \backslash \bar{\Omega}}=0$, and the rest of the proof is as in Theorem 1 . 
Clearly, (5.4) implies that

$$
\left(\Delta-\kappa_{j}^{2}\right) \vartheta_{j}=\vartheta_{j-1}, \quad \text { for } j=2, \ldots, m, \quad \text { and }\left(\Delta-\kappa_{1}^{2}\right) \vartheta_{1}=\nu,
$$

in the sense of distributions. Also, $\left.\vartheta_{1}\right|_{\partial \Omega^{\prime}}=\cdots=\left.\vartheta_{m}\right|_{\partial \Omega^{\prime}}=0$, since

$$
\vartheta_{j}(\boldsymbol{x})=\nu\left(\tau_{\boldsymbol{x}} \varphi_{j}\right)=\nu\left(\sum_{\ell=1}^{j} \beta_{\ell} \tau_{\boldsymbol{x}} e_{1}\left(\cdot ; \kappa_{\ell}^{2}\right)\right)=\sum_{\ell=1}^{j} \beta_{\ell} \nu\left(\tau_{\boldsymbol{x}} e_{1}\left(\cdot ; \kappa_{\ell}^{2}\right)\right)=0 .
$$

As in the proof of Theorem [5, we obtain $\left.\vartheta_{1}\right|_{\mathbb{R}^{n} \backslash \bar{\Omega}}=0$. Therefore, $\left(\Delta-\kappa_{2}^{2}\right) \vartheta_{2}=0$ in $\mathbb{R}^{n} \backslash \bar{\Omega}$. Since $\left.\vartheta_{2}\right|_{\partial \Omega^{\prime}}=0$, then, using again Theorem $\left[5\right.$, we obtain $\left.\vartheta_{2}\right|_{\mathbb{R}^{n} \backslash \bar{\Omega}}=0$, and, inductively, that $\left.\vartheta_{m}\right|_{\mathbb{R}^{n} \backslash \bar{\Omega}}=0$.

If $n=2$ and $\kappa_{1}=0$, then, as in the proof of Theorem $1,\left.\vartheta_{1}\right|_{\mathbb{R}^{2} \backslash \bar{\Omega}}=0$, provided that the constants are included in $\mathcal{X}$ and the rest of the proof is identical.

\section{Remark 5.1.}

(i) The function $e_{m}\left(\cdot ; \kappa^{2}\right)$ given by (5.9) is a fundamental solution of the operator $\mathcal{L}=\left(\Delta-\kappa^{2}\right)^{m}$, where $\kappa>0$. With $\Omega, \Omega^{\prime}$ and $\boldsymbol{y}_{i}$ as in Theorem [6, one can show, following the lines of the proof of Theorem [6, that linear combinations of the form

$$
u^{N}(\boldsymbol{x})=\sum_{i=1}^{N} \sum_{j=1}^{m} c_{i j} e_{j}\left(\boldsymbol{x}-\boldsymbol{y}_{i}, \kappa^{2}\right),
$$

are dense in $\mathcal{Y}_{\ell}=\left\{u \in C^{2 m}(\Omega): \mathcal{L} u=0\right.$ in $\left.\Omega\right\} \cap C^{\ell}(\bar{\Omega})$ with respect to the norm of $C^{\ell}(\bar{\Omega})$.

(ii) More generally, the fundamental solution of the operator

$$
\mathcal{L}=\left(\Delta-\kappa_{1}^{2}\right)^{\nu_{1}} \cdots\left(\Delta-\kappa_{m}^{2}\right)^{\nu_{m}},
$$

given by (5.8), is a linear combination of the functions $e_{\ell}\left(\cdot ; \kappa_{j}^{2}\right), j=1, \ldots, m$, $\ell=1, \ldots, \nu_{j}$. A density result, analogous to Theorem [6 with linear combinations of the form

$$
u^{N}(\boldsymbol{x})=\sum_{i=1}^{N} \sum_{j=1}^{m} \sum_{\ell=1}^{\nu_{j}} c_{i j \ell} e_{\ell}\left(\boldsymbol{x}-\boldsymbol{y}_{i}, \kappa_{j}^{2}\right)
$$

holds for the solutions of the equation $\mathcal{L} u=0$.

(iii) In particular, if for every $j=1, \ldots, m$ the linear space $\mathcal{X}^{j, \nu_{j}}$ is dense in

$$
\mathcal{Y}_{\ell}^{j, \nu_{j}}=\left\{u \in C^{2 \nu_{j}}(\Omega):\left(\Delta-\kappa_{j}^{2}\right)^{\nu_{j}} u=0 \text { in } \Omega\right\} \cap C^{\ell}(\bar{\Omega}),
$$

then $\mathcal{X}^{1, \nu_{1}} \oplus \cdots \oplus \mathcal{X}^{m, \nu_{m}}$ is dense in $\mathcal{Y}_{\ell}$ given by

$$
\mathcal{Y}_{\ell}=\left\{u \in C^{2 \nu_{1}+\cdots+2 \nu_{m}}(\Omega): \prod_{j=1}^{m}\left(\Delta-\kappa_{j}^{2}\right)^{\nu_{j}} u=0 \text { in } \Omega\right\} \cap C^{\ell}(\bar{\Omega}),
$$

whenever $k_{1}^{2}, \ldots, k_{m}^{2}$ are distinct complex numbers. 


\section{Applications of the MFS to elliptic systems}

6.1. The MFS for systems of partial differential equations. Fundamental solutions are also defined for systems of partial differential equations and the MFS has been applied for the solution of boundary value problems in which the corresponding equations constitute an elliptic system. Let $\mathcal{L} \boldsymbol{u}=\mathbf{0}$ be a $d \times d$ linear homogeneous system with

$$
\mathcal{L} \boldsymbol{u}=\left(\begin{array}{ccc}
\mathcal{L}_{11} & \cdots & \mathcal{L}_{1 d} \\
\vdots & & \vdots \\
\mathcal{L}_{d 1} & \cdots & \mathcal{L}_{d d}
\end{array}\right)\left(\begin{array}{c}
u_{1} \\
\vdots \\
u_{d}
\end{array}\right)=\left(\begin{array}{c}
\sum_{j=1}^{d} \mathcal{L}_{1 j} u_{j} \\
\vdots \\
\sum_{j=1}^{d} \mathcal{L}_{d j} u_{j}
\end{array}\right),
$$

where $\boldsymbol{u}=\left(u_{1}, \ldots, u_{d}\right) \in \mathbb{R}^{d}, \mathcal{L}=\left(\mathcal{L}_{i j}\right)_{i, j=1}^{d}$ and $\mathcal{L}_{i j}=\sum_{|\alpha| \leq m} a_{\alpha}^{i j} D^{\alpha}$ are scalar partial differential operators in $\mathbb{R}^{n}$ with constant coefficients. Alternatively, $\mathcal{L}=\sum_{|\alpha| \leq m} A_{\alpha} D^{\alpha}$, where $A_{\alpha}=\left(a_{\alpha}^{i j}\right)_{i, j=1}^{d}$ are constant matrices. A fundamental solution of $\mathcal{L}$ is a matrix $E=\left(e_{i j}\right)_{i, j=1}^{d}$, where $e_{i j}$ are real-valued functions, smooth in $\mathbb{R}^{n} \backslash\{0\}$, satisfying $\mathcal{L} E=\delta I$, in the sense of distributions, where $\delta$ is the Dirac measure with unit mass at the origin and $I$ is the identity matrix in $\mathbb{R}^{d \times d}$. This means that

$$
\sum_{j=1}^{d} \mathcal{L}_{i j} e_{j k}=\left\{\begin{array}{lll}
\delta & \text { if } & i=k \\
0 & \text { if } & i \neq k
\end{array}\right.
$$

or equivalently,

$$
\int_{\mathbb{R}^{n}} E(\boldsymbol{x}) \mathcal{L}^{\star} \boldsymbol{\psi}(\boldsymbol{x}) d \boldsymbol{x}=\boldsymbol{\psi}(\mathbf{0})
$$

for every $\boldsymbol{x} \in \mathbb{R}^{n}$ and $\boldsymbol{\psi}=\left(\psi_{1}, \ldots, \psi_{d}\right)$ with $\psi_{i} \in C_{0}^{\infty}\left(\mathbb{R}^{n}\right)$, where $\mathcal{L}^{\star}$ is the adjoint operator of $\mathcal{L}$. If $f_{1}, \ldots, f_{d}$ are measurable functions and

$$
u_{i}(\boldsymbol{x})=\sum_{j=1}^{d} \int_{\mathbb{R}^{n}} e_{i j}(\boldsymbol{x}-\boldsymbol{y}) f_{j}(\boldsymbol{y}) d \boldsymbol{y}, \quad i=1, \ldots, d,
$$

then $\mathcal{L} \boldsymbol{u}=\boldsymbol{f}$, where $\boldsymbol{f}=\left(f_{1}, \ldots, f_{d}\right)$ and $\boldsymbol{u}=\left(u_{1}, \ldots, u_{d}\right)$, provided that the integrals on the right-hand side of (6.3) are meaningful. Formulae (6.3) can be written in vector form as

$$
\boldsymbol{u}(\boldsymbol{x})=\int_{\mathbb{R}^{n}} E(\boldsymbol{x}-\boldsymbol{y}) \boldsymbol{f}(\boldsymbol{y}) d \boldsymbol{y},
$$

or even simpler as $\boldsymbol{u}=E * \boldsymbol{f}$.

In the MFS for second order elliptic systems, the components of the approximate solution $\boldsymbol{u}^{N}=\left(u_{1}^{N}, \ldots, u_{d}^{N}\right)$ are linear combinations of the form

$$
u_{i}^{N}(\boldsymbol{x})=\sum_{k=1}^{d} \sum_{j=1}^{N} c_{j}^{k} e_{i k}\left(\boldsymbol{x}-\boldsymbol{y}_{j}\right), \quad i=1, \ldots, d,
$$

with $\left\{\boldsymbol{y}_{j}\right\}_{j=1}^{N}$, the singularities, lying on a given pseudo-boundary (see Kupradze and Aleksidze [33]). Alternatively, (6.4) can be written as

$$
\boldsymbol{u}^{N}(\boldsymbol{x})=\sum_{j=1}^{N} E\left(\boldsymbol{x}-\boldsymbol{y}_{j}\right) \boldsymbol{c}_{j}
$$


where $\boldsymbol{c}_{j}=\left(c_{j}^{1}, \ldots, c_{j}^{d}\right)$, or equivalently

$$
\boldsymbol{u}^{N}(\boldsymbol{x})=\sum_{k=1}^{d} \sum_{j=1}^{N} c_{j}^{k} \boldsymbol{e}_{k}\left(\boldsymbol{x}-\boldsymbol{y}_{j}\right)
$$

with $\boldsymbol{e}_{k}, k=1, \ldots, d$, the columns of the matrix $E$.

In the case of systems, the density results we seek are with respect to the norms of the spaces $C^{\ell}\left(\bar{\Omega} ; \mathbb{R}^{d}\right)$ which contain vector-valued functions $\boldsymbol{u}=\left(u_{1}, \ldots, u_{d}\right)$ : $\bar{\Omega} \rightarrow \mathbb{R}^{d}$, for which $u_{i} \in C^{\ell}(\bar{\Omega}), i=1, \ldots, d$. The norm of $C^{\ell}\left(\bar{\Omega} ; \mathbb{R}^{d}\right)$ is defined as

$$
|\boldsymbol{u}|_{\ell}=\max _{i=1, \ldots, d}\left|u_{i}\right|_{\ell}
$$

Analogously, $C^{\ell}\left(\Omega ; \mathbb{R}^{d}\right)$ is the space of vector-valued functions $\boldsymbol{u}: \Omega \rightarrow \mathbb{R}^{d}$, where $\boldsymbol{u}=\left(u_{1}, \ldots, u_{d}\right)$ and $u_{i} \in C^{\ell}(\Omega), i=1, \ldots, d$. If $\boldsymbol{\nu}$ is an element of the dual of $C^{\ell}\left(\bar{\Omega} ; \mathbb{R}^{d}\right)$, then $\boldsymbol{\nu}$ can be represented as

$$
\boldsymbol{\nu}(\boldsymbol{u})=\sum_{i=1}^{d} \nu_{i}\left(u_{i}\right), \quad \boldsymbol{u} \in C^{\ell}\left(\bar{\Omega} ; \mathbb{R}^{d}\right),
$$

where $\nu_{1}, \ldots, \nu_{d} \in\left(C^{\ell}(\bar{\Omega})\right)^{\prime}$.

The set of test functions on $\Omega$ with values in $\mathbb{R}^{d}$ is denoted by $\mathscr{D}\left(\Omega ; \mathbb{R}^{d}\right)$ and its dual by $\mathscr{D}^{\prime}\left(\Omega ; \mathbb{R}^{d}\right)$. If $\boldsymbol{T} \in \mathscr{D}^{\prime}\left(\Omega ; \mathbb{R}^{d}\right)$, then there exist $T_{1}, \ldots, T_{d} \in \mathscr{D}^{\prime}(\Omega)$ such that

$$
\boldsymbol{T}(\boldsymbol{\psi})=T_{1}\left(\psi_{1}\right)+\cdots+T_{d}\left(\psi_{d}\right), \quad \text { where } \quad \boldsymbol{\psi}=\left(\psi_{1}, \ldots, \psi_{d}\right) \in \mathscr{D}\left(\Omega ; \mathbb{R}^{d}\right) .
$$

If $\boldsymbol{\nu}$ is a distribution with compact support, and $E=E(\boldsymbol{x})$ is a fundamental solution of the operator $\mathcal{L}$, then the convolution $\boldsymbol{\vartheta}=E * \boldsymbol{\nu}$ defines a distribution in $\mathscr{D}^{\prime}\left(\mathbb{R}^{n} ; \mathbb{R}^{d}\right)$ according to

$$
\boldsymbol{\vartheta}(\boldsymbol{\psi})=\left(\boldsymbol{e}_{1} * \boldsymbol{\nu}\right)\left(\psi_{1}\right)+\cdots+\left(\boldsymbol{e}_{d} * \boldsymbol{\nu}\right)\left(\psi_{d}\right)=\sum_{i=1}^{d} \sum_{j=1}^{n}\left(e_{j i} * \nu_{j}\right)\left(\psi_{i}\right),
$$

where $\boldsymbol{e}_{1}, \ldots, \boldsymbol{e}_{d}$ are the columns of $E$ and $\boldsymbol{\psi}=\left(\psi_{1}, \ldots, \psi_{d}\right) \in \mathscr{D}\left(\mathbb{R}^{n} ; \mathbb{R}^{d}\right)$. Also, $\mathcal{L} \vartheta=\boldsymbol{\nu}$ in the sense of distributions, i.e.,

$$
(\mathcal{L} \boldsymbol{\vartheta})(\boldsymbol{\psi})=\boldsymbol{\vartheta}\left(\mathcal{L}^{\star} \boldsymbol{\psi}\right)=\boldsymbol{\nu}(\boldsymbol{\psi}) \text { for every } \quad \boldsymbol{\psi} \in \mathscr{D}\left(\mathbb{R}^{n} ; \mathbb{R}^{d}\right) .
$$

A partial differential operator of the form $\mathcal{L}=\sum_{|\alpha|<m} A_{\alpha} D^{\alpha}$, where $A_{\alpha}$ are constant $d \times d$ matrices, is said to be elliptic if their principal symbol

$$
\sigma(\mathcal{L})(\boldsymbol{\xi})=\sum_{|\alpha|=m} \boldsymbol{\xi}^{\alpha} A_{\alpha}
$$

is a nonsingular matrix in $\mathbb{R}^{d \times d}$ for every $\boldsymbol{\xi}=\left(\xi_{1}, \ldots, \xi_{n}\right) \in \mathbb{R}^{n} \backslash\{\mathbf{0}\}$, where $\alpha=$ $\left(\alpha_{1}, \ldots, \alpha_{n}\right) \in \mathbb{N}^{n}$ and $\boldsymbol{\xi}^{\alpha}=\xi_{1}^{\alpha_{1}} \ldots \xi_{n}^{\alpha_{n}}$. The solutions of homogeneous elliptic systems with constant coefficients (more generally, with analytic coefficients) are real analytic functions. (See [52, Example 4.3.4].)

Remark 6.1. The MFS for systems was introduced in [33]. Since then, several formulations of the method, in numerous applications, have been used. See [17. 


\subsection{A linear model in the theory of elastostatics.}

6.2.1. The Cauchy-Navier equations. The governing equations of equilibrium for a homogeneous, isotropic, linear-elastic solid $\Omega \subset \mathbb{R}^{3}$, in the absence of body forces, are the Cauchy-Navier equations

$$
(\lambda+\mu) \sum_{k=1}^{3} \frac{\partial^{2}}{\partial x_{k} \partial x_{i}} u_{k}+\mu \frac{\partial^{2}}{\partial x_{k}^{2}} u_{i}=0, \quad i=1,2,3, \quad \text { in } \Omega,
$$

where $u_{1}, u_{2}$ and $u_{3}$ denote the displacements and $\lambda, \mu$ are real constants known as the Lamé parameters. The Cauchy-Navier system can be alternatively written as $\Delta^{*} \boldsymbol{u}=0$, where

$$
\Delta^{*}=\mu \Delta+(\lambda+\mu) \operatorname{grad} \operatorname{div},
$$

and $\boldsymbol{u}=\left(u_{1}, u_{2}, u_{3}\right)$. Clearly, $\Delta^{*}$ is a self-adjoint operator, i.e., $\left(\Delta^{*}\right)^{\star}=\Delta^{*}$. System (6.5) is elliptic if $\mu>0$ and $\lambda+\mu \geq 0$. Dirichlet boundary conditions

$$
u_{i}=f_{i}, \quad i=1,2,3, \quad \text { on } \partial \Omega,
$$

guarantee uniqueness for the solutions of (6.5). This is a consequence of Betti's second formula 32

$$
\int_{\Omega} \boldsymbol{u} \cdot \Delta^{*} \boldsymbol{u} d \boldsymbol{x}=\int_{\partial \Omega} \boldsymbol{u} \cdot T \boldsymbol{\nu} d s-\int_{\Omega} \mathcal{B}(\boldsymbol{u}, \boldsymbol{u}) d \boldsymbol{x},
$$

where $\boldsymbol{\nu}$ is the unit exterior normal,

$$
\mathcal{B}(\boldsymbol{u})=2 \mu \sum_{k, \ell=1}^{3} u_{k \ell}^{2}+\lambda(\operatorname{div} \boldsymbol{u})^{2},
$$

with $u_{k \ell}=\frac{1}{2}\left(\frac{\partial u_{k}}{\partial x_{\ell}}+\frac{\partial u_{\ell}}{\partial x_{k}}\right)$ and $T=\left(\tau_{k \ell}\right)_{k, \ell=1}^{3}$ is the stress tensor with

$$
\tau_{k \ell}=\left\{\begin{array}{lll}
\lambda \operatorname{div} \boldsymbol{u}+2 \mu \frac{\partial u_{k}}{\partial x_{k}} & \text { if } \quad k=\ell, \\
2 \mu u_{k \ell} & \text { if } \quad k \neq \ell .
\end{array}\right.
$$

Remark 6.2. Formula (6.7) guarantees uniqueness for the exterior problem, provided that $(32,46])$

$$
\lim _{|\boldsymbol{x}| \rightarrow \infty} \boldsymbol{u}(\boldsymbol{x})=0 \quad \text { and } \quad \varrho \frac{\partial \boldsymbol{u}}{\partial \varrho}=o(1)
$$

when $\varrho=|\boldsymbol{x}|$ is large.

Also, Betti's second formula guarantees uniqueness, up to additive constants, to the boundary value problem of Cauchy-Navier equations with natural conditions, i.e., conditions of Neumann type prescribing the stresses on the boundary, which are of the form

$$
\lambda(\operatorname{div} \boldsymbol{u}) \boldsymbol{\nu}+2 \mu \frac{\partial \boldsymbol{u}}{\partial \boldsymbol{\nu}}+\mu \boldsymbol{\nu} \times \operatorname{curl} \boldsymbol{u}=\boldsymbol{p},
$$

where $\boldsymbol{\nu}$ is the unit external normal and $\boldsymbol{p}$ is the pressure. 
6.2.2. Approximation by fundamental solutions. The matrix

$$
E(\boldsymbol{x})=\left(e_{i j}(\boldsymbol{x})\right)_{i, j=1}^{3}=-\frac{1}{8 \pi \mu(2 \mu+\lambda)}\left(\frac{3 \mu+\lambda}{|\boldsymbol{x}|} I+\frac{\mu+\lambda}{|\boldsymbol{x}|^{3}} \boldsymbol{x} \cdot \boldsymbol{x}^{\boldsymbol{T}}\right)
$$

is a fundamental solution of $\Delta^{*}$, where $\boldsymbol{x} \cdot \boldsymbol{x}^{T}=\left(x_{i} x_{j}\right)_{i, j=1}^{3} \in \mathbb{R}^{3}$. The expression (6.8) is due to Lord Kelvin (see [36). For further details and the derivation of (6.8), see 35 .

We have the following density result:

Theorem 7. Let $\Omega, \Omega^{\prime}$ be open bounded domains in $\mathbb{R}^{3}$, with $\Omega$ satisfying the segment condition and $\Omega^{\prime}$ with smooth boundary and embracing $\Omega$. Also, let $\ell$ be a nonnegative integer. Then the space $\mathcal{X}$ of finite linear combinations of the form $\sum_{j=1}^{N} E\left(\boldsymbol{x}-\boldsymbol{y}_{j}\right) \boldsymbol{c}_{j}$, where $E(\boldsymbol{x})$ is given by (6.8) and $\left\{\boldsymbol{y}_{j}\right\}_{j=1}^{N}$ lie on $\partial \Omega^{\prime}$, is dense in

$$
\mathcal{Y}_{\ell}=\left\{\boldsymbol{u} \in C^{2}\left(\Omega ; \mathbb{R}^{3}\right) \text { satisfying (6.5) in } \Omega\right\} \cap C^{\ell}\left(\bar{\Omega} ; \mathbb{R}^{3}\right)
$$

with respect to the norm of $C^{\ell}\left(\bar{\Omega} ; \mathbb{R}^{3}\right)$.

Sketch of proof. As in the proof of Theorem 1, we need to show that whenever a functional $\boldsymbol{\nu}$ in $\left(C^{\ell}\left(\bar{\Omega} ; \mathbb{R}^{3}\right)\right)^{\prime}$ annihilates $\mathcal{X}$, then $\boldsymbol{\nu}$ annihilates $\mathcal{Y}_{\ell}$ as well. Assume $\boldsymbol{\nu} \in\left(C^{\ell}(\bar{\Omega})\right)^{\prime}$ annihilates $\mathcal{X}$. Then, in particular, $\boldsymbol{\nu}\left(\tau_{\boldsymbol{y}} E \boldsymbol{c}\right)=0$, for every $\boldsymbol{y} \in \partial \Omega^{\prime}$ and $\boldsymbol{c} \in \mathbb{R}^{3}$, and consequently $\vartheta_{i}(\boldsymbol{y})=\boldsymbol{\nu}\left(\tau_{\boldsymbol{y}} \boldsymbol{e}_{i}\right)=0$, for $i=1,2,3$, and for every $\boldsymbol{y} \in \partial \Omega^{\prime}$, where $\boldsymbol{e}_{1}, \boldsymbol{e}_{2}, \boldsymbol{e}_{3}$ are the columns of $\boldsymbol{E}$. Clearly, $\boldsymbol{\vartheta}=\left(\vartheta_{1}, \vartheta_{2}, \vartheta_{3}\right)$ is a smooth function and, in fact, $\Delta^{*} \boldsymbol{\vartheta}=0$ in $\mathbb{R}^{3} \backslash \bar{\Omega}$. One can then show that, due to (6.7), $\vartheta$ vanishes in $\mathbb{R}^{3} \backslash \bar{\Omega}$. Note that, as in the proof of Theorem 1, we need to study separately the unbounded and the bounded connected components of $\mathbb{R}^{3} \backslash \bar{\Omega}$. Meanwhile, $\boldsymbol{\vartheta}$ is defined in the whole of $\mathbb{R}^{3}$ as a distribution $(\boldsymbol{\vartheta}=E * \boldsymbol{\nu})$, and satisfies $\Delta^{*} \boldsymbol{\vartheta}=\Delta^{*}(E * \boldsymbol{\nu})=\boldsymbol{\nu}$, in the sense of distributions. A suitable version of Lemma 1 for systems yields a sequence $\left\{\boldsymbol{\psi}_{k}\right\}_{k \in \mathbb{N}} \in C_{0}^{\infty}\left(\Omega ; \mathbb{R}^{3}\right)$ for which $\left\{\Delta^{*} \boldsymbol{\psi}_{\boldsymbol{k}}\right\}_{k \in \mathbb{N}}$ converges to $\boldsymbol{\nu}$ in the weak ${ }^{\star}$ sense of $C^{\ell}\left(\bar{\Omega} ; \mathbb{R}^{3}\right)$. Thus, for $\boldsymbol{u} \in \mathcal{Y}_{\ell}$, we have

$$
\boldsymbol{\nu}(\boldsymbol{u})=\lim _{k \rightarrow \infty} \int_{\mathbb{R}^{n}} \Delta^{*} \boldsymbol{\psi}_{k} \cdot \boldsymbol{u} d \boldsymbol{x}=\lim _{k \rightarrow \infty} \int_{\mathbb{R}^{n}} \boldsymbol{\psi}_{k} \cdot \Delta^{*} \boldsymbol{u} d \boldsymbol{x}=\mathbf{0},
$$

since $\Delta^{*}$ is self-adjoint.

Remark 6.3. A similar density result is obtainable in the two-dimensional version of the Cauchy-Navier system, where a fundamental solution is given by

$$
E(\boldsymbol{x})=-\frac{1}{4 \pi \mu(\lambda+2 \mu)}\left((\lambda+3 \mu) \log |\boldsymbol{x}| I-\frac{\lambda+\mu}{|\boldsymbol{x}|^{2}} \boldsymbol{x} \cdot \boldsymbol{x}^{T}\right),
$$

provided that the constant functions are included in the space $\mathcal{X}$ of the linear combinations of the columns of $E$ with singularities on the pseudo-boundary.

\subsection{Equations of the static theory of thermo-elasticity.}

6.3.1. The three-dimensional model. The displacements $\boldsymbol{u}=\left(u_{1}, u_{2}, u_{3}\right)$ and the temperature $\vartheta$ of a thermo-elastic medium are described by the system (see [34])

$$
\begin{aligned}
\Delta^{*} \boldsymbol{u} & =\gamma \operatorname{grad} \vartheta, \\
\Delta \vartheta & =0,
\end{aligned}
$$

where $\gamma$ is positive constant. Equations (6.10) constitute a $4 \times 4$ elliptic system with unknowns $\boldsymbol{u}$ and $\vartheta$. It is readily seen that the corresponding Dirichlet problem for 
bounded domains, in which the displacements and the temperature are prescribed on the boundary, enjoys uniqueness.

A fundamental solution $G=\left(g_{i j}\right)_{i, j=1}^{4}$ of (6.10) is given by (see [4])

$g_{i j}(\boldsymbol{x})=-\frac{\left(1-\delta_{i 4}\right)\left(1-\delta_{j 4}\right)}{8 \pi \mu(\lambda+2 \mu)}\left((\lambda+\mu) \frac{x_{i} x_{j}}{|\boldsymbol{x}|^{3}}+(\lambda+3 \mu) \frac{\delta_{i j}}{|\boldsymbol{x}|}\right)-\frac{\gamma \delta_{j 4}\left(1-\delta_{i 4}\right)}{8 \pi(\lambda+2 \mu)} \frac{x_{i}}{|\boldsymbol{x}|}-\frac{\delta_{i 4} \delta_{j 4}}{4 \pi|\boldsymbol{x}|}$,

$i, j=1,2,3,4$, where $\delta_{i j}$ is the symbol of Kronecker. The matrix $G$ can be alternatively written in a block form as

$$
G(\boldsymbol{x})=\left(\begin{array}{c|r}
E(\boldsymbol{x}) & \eta \frac{\boldsymbol{x}}{|\boldsymbol{x}|} \\
\hline 0 & -e_{1}(\boldsymbol{x})
\end{array}\right)
$$

where $E(\boldsymbol{x})$ is the fundamental solution of the operator $\Delta^{*}$ given by (6.8), $\eta=$ $-\frac{\gamma}{8 \pi(\lambda+2 \mu)}$ and $e_{1}$ is the fundamental solution of $-\Delta$ given by (1.6).

6.3.2. Approximation of the solutions of (6.10). The temperature $\vartheta$ is a harmonic function and it can be thus approximated by linear combinations of the form

$$
\vartheta^{N}(\boldsymbol{x})=\sum_{k=1}^{N} a_{k} e_{1}\left(\boldsymbol{x}-\boldsymbol{y}_{k}\right),
$$

where $\left\{\boldsymbol{y}_{k}\right\}_{k=1}^{N}$ lie on a prescribed pseudo-boundary $\partial \Omega^{\prime},\left\{a_{k}\right\}_{k=1}^{N}$ are real constants. Equation (6.10a now becomes

$$
\Delta^{*} \boldsymbol{u}=\gamma \operatorname{grad} \sum_{k=1}^{N} a_{k} e_{1}\left(\boldsymbol{x}-\boldsymbol{y}_{k}\right)=-\frac{\gamma}{4 \pi} \sum_{k=1}^{N} a_{k} \frac{\boldsymbol{x}-\boldsymbol{y}_{k}}{\left|\boldsymbol{x}-\boldsymbol{y}_{k}\right|^{3}},
$$

which are inhomogeneous. It is straightforward that

$$
\Delta^{*}\left(\frac{\boldsymbol{x}}{|\boldsymbol{x}|}\right)=-2(\lambda+2 \mu) \frac{\boldsymbol{x}}{|\boldsymbol{x}|^{3}},
$$

which allows us to obtain a particular solution $\boldsymbol{u}_{p}$ of (6.13):

$$
\boldsymbol{u}_{p}(\boldsymbol{x})=\frac{\gamma}{8 \pi(\lambda+2 \mu)} \sum_{k=1}^{N} a_{k} \frac{\boldsymbol{x}-\boldsymbol{y}_{k}}{\left|\boldsymbol{x}-\boldsymbol{y}_{k}\right|} .
$$

Clearly, $\Delta^{*}\left(\boldsymbol{u}-\boldsymbol{u}_{p}\right)=0$, and by virtue of Theorem 7 the difference $\boldsymbol{v}=\boldsymbol{u}-\boldsymbol{u}_{p}$ can be approximated by linear combinations of the form

$$
\boldsymbol{v}^{M}(\boldsymbol{x})=\sum_{j=1}^{M} E\left(\boldsymbol{x}-\boldsymbol{z}_{j}\right) \boldsymbol{b}_{j},
$$

where $E(\boldsymbol{x})$ is given by (6.8), the points $\left\{\boldsymbol{z}_{j}\right\}_{j=1}^{M}$ also lie on $\partial \Omega^{\prime}$ and $\left\{\boldsymbol{b}_{j}\right\}_{j=1}^{M}$ are constant vectors in $\mathbb{R}^{3}$. Altogether we have the following approximate solution:

$$
\begin{aligned}
& \boldsymbol{u}^{M, N}(\boldsymbol{x})=\sum_{j=1}^{M} E\left(\boldsymbol{x}-\boldsymbol{z}_{j}\right) \boldsymbol{b}_{j}+\frac{\gamma}{8 \pi(\lambda+2 \mu)} \sum_{k=1}^{N} a_{k} \frac{\boldsymbol{x}-\boldsymbol{y}_{k}}{\left|\boldsymbol{x}-\boldsymbol{y}_{k}\right|} \\
& \vartheta^{M, N}(\boldsymbol{x})=\vartheta^{N}(\boldsymbol{x})=\sum_{k=1}^{N} a_{k} e_{1}\left(\boldsymbol{x}-\boldsymbol{y}_{k}\right) .
\end{aligned}
$$


If we set $\left\{\boldsymbol{x}_{\ell}\right\}_{\ell=1}^{L}=\left\{\boldsymbol{y}_{k}\right\}_{k=1}^{N} \cup\left\{\boldsymbol{z}_{j}\right\}_{j=1}^{M}$, the vector $\left(u_{1}^{M, N}, u_{2}^{M, N}, u_{3}^{M, N}, \vartheta^{M, N}\right)$ is a linear combination of the columns of the matrices $G\left(\boldsymbol{x}-\boldsymbol{x}_{m}\right), m=1, \ldots, L$.

We have the following density result. (For a detailed proof see [46].)

Theorem 8. Let $\Omega, \Omega^{\prime}$ be open and bounded domains in $\mathbb{R}^{3}$, with $\Omega$ satisfying the segment condition and $\Omega^{\prime}$ with smooth boundary and embracing $\Omega$. Also, let $\ell$ be a nonnegative integer. Then the space $\mathcal{X}$ of finite linear combinations of the form $\sum_{j=1}^{N} G\left(\boldsymbol{x}-\boldsymbol{y}_{j}\right) \boldsymbol{c}_{j}$, where $G(\boldsymbol{x})$ is given by (6.12), $\left\{\boldsymbol{y}_{j}\right\}_{j=1}^{N}$ lie on $\partial \Omega^{\prime}$ and $\boldsymbol{c}_{j}$ are constant vectors in $\mathbb{R}^{4}$, is dense in

$$
\mathcal{Y}_{\ell}=\left\{\left(u_{1}, u_{2}, u_{3}, \vartheta\right) \in C^{2}\left(\Omega ; \mathbb{R}^{4}\right) \text { satisfying (6.10) in } \Omega\right\} \cap C^{\ell}\left(\bar{\Omega} ; \mathbb{R}^{4}\right)
$$

with respect to the norm of $C^{\ell}\left(\bar{\Omega} ; \mathbb{R}^{4}\right)$.

\section{Concluding Remarks}

We have extended previous density results for solutions of elliptic partial differential equations and elliptic systems by finite linear combinations of their fundamental solutions, the singularities of which lie on a prescribed pseudo-boundary. In particular, we have proved a lemma which allows us to establish the density of linear combinations of fundamental solutions with respect to the norms of the spaces $C^{\ell}(\bar{\Omega})$. A slight modification of this lemma provides $C^{\ell, \sigma}$-density results ([4]). In our density results, the domains may possess holes and their boundaries are required to satisfy a rather weak condition, the segment condition. Meanwhile, we observed that analogous density results are not obtainable in the spaces $W^{k, \infty}(\Omega)$ and $\Lambda^{\ell, \sigma}(\bar{\Omega})$.

Using our approach, we proved that the finite linear combinations of the fundamental solutions of the Laplacian and $m$-harmonic operators are dense, with respect to any $C^{\ell}$-norm. In the two-dimensional case we observed that linear combinations of fundamental solutions of Laplace's equation with singularities on a prescribed pseudo-boundary, are not always dense in the space of harmonic functions. However, if the pseudo-boundary is a subset of a unit disk, then such linear combinations are dense in the space of harmonic functions. This fact allows us to propose an alternative MFS formulation with rescaled fundamental solutions. In the case of an analytic pseudo-boundary $\partial \Omega^{\prime}$, we showed that the same density results hold even if the locations of the singularities are restricted to an open subset of $\partial \Omega^{\prime}$. We also propose alternative MFS formulations for the approximation of the solutions of the $m$-harmonic equation which exploit Almansi's representation. Analogous density results are obtained for the solutions of the modified Helmholtz equation and equations of the form $\mathcal{L} u=\left(\Delta-\kappa_{1}^{2}\right)^{\nu_{1}} \cdots\left(\Delta-\kappa_{m}^{2}\right)^{\nu_{k}} u$. Finally, we extended our density results to elliptic systems and obtained similar results for the Cauchy-Navier system and a system in the linear theory of thermo-elastostatics.

The MFS has been applied, with very satisfactory numerical results, to a variety of boundary value problems in which the corresponding density results have not been established yet. Such problems include the classical formulations for the Helmholtz equation, the Navier system in the theory of linear elasticity and Maxwell equations ([4, 17, 32]). Also, questions regarding the applicability of the MFS for mixed, external and contact problems remain unanswered.

Unfortunately, density results do not guarantee the efficiency of any MFS algorithm for the solution of the corresponding boundary value problem. Convergence 
and error estimates for MFS algorithms are available only for very specific domains, operators and distributions of singularities.

\section{ACKNOWLEDGEMENTS}

The author wishes to thank Professors G. Akrivis, C. S. Chen, G. Fairweather, L. Grafakos, A. Karageorghis and E. Samiou for the illuminating conversations he had with them and the anonymous referees for their instructive comments. In particular, the author wishes to thank C. Arvanitis for his assistance in technical matters in the preparation of this paper.

\section{APPENDix}

Proof of Lemma 1. Since $\Omega$ satisfies the segment condition, then for every $\boldsymbol{x} \in \partial \Omega$, there exist a vector $\boldsymbol{\xi}_{\boldsymbol{x}} \in \mathbb{R}^{n} \backslash\{0\}$ and an open neighborhood $U_{\boldsymbol{x}}$ of $\boldsymbol{x}$, such that, if $\boldsymbol{y} \in U_{\boldsymbol{x}} \cap \bar{\Omega}$, then $\boldsymbol{y}+\varepsilon \boldsymbol{\xi}_{\boldsymbol{x}} \in \Omega$, for every $\varepsilon \in(0,1)$. Since $\partial \Omega$ is compact, there is a finite collection of such neighborhoods $\left\{U_{j}\right\}_{j=1}^{J}$ covering $\partial \Omega$. The collection $\left\{U_{j}\right\}_{j=1}^{J}$ becomes an open cover of $\bar{\Omega}$ by adding a suitable open set $U_{0}$, which can be chosen so that $\bar{U}_{0} \subset \Omega$. We denote by $\boldsymbol{\xi}_{j}$ the nonzero vector corresponding to $U_{j}$, when $j=1, \ldots, J$, and set $\boldsymbol{\xi}_{0}=0$. (We have that $\tau_{\varepsilon \boldsymbol{\xi}_{j}}\left[U_{j} \cap \bar{\Omega}\right] \subset \Omega$, for every $\varepsilon \in(0,1)$ and $j=0, \ldots, J$.) Let $\left\{\psi^{j}\right\}_{j=0}^{J}$ be an infinitely differentiable partition of unity corresponding to the covering $\left\{U_{j}\right\}_{j=0}^{J}$ of $\bar{\Omega}$, i.e., $\psi_{j} \in \mathscr{D}\left(U_{j}\right)$ and $\sum_{j=0}^{J} \psi_{j}(\boldsymbol{x})=1$, for every $\boldsymbol{x} \in \bar{\Omega}$.

Next, we set $\nu^{j}=\mathcal{L}\left(\psi^{j}(e * \nu)\right), j=0, \ldots, J$, which provides a decomposition of $\nu$ as

$$
\sum_{j=0}^{J} \nu^{j}=\sum_{j=0}^{J} \mathcal{L}\left(\psi^{j}(e * \nu)\right)=\mathcal{L}\left(\left(\sum_{j=0}^{J} \psi^{j}\right) e * \nu\right)=\mathcal{L}(e * \nu)=\nu .
$$

It is clear that, $\operatorname{supp} \nu^{j} \subset \bar{U}_{j} \cap \bar{\Omega}$. In the rest of the proof we shall first show that $\nu^{j} \in\left(C^{\ell}(\bar{\Omega})\right)^{\prime}$. Then, we define

$$
\vartheta_{j, \varepsilon}=\tau_{\varepsilon \boldsymbol{\xi}_{j}} \circ\left(\psi^{j} \vartheta\right) \quad \text { and } \quad \nu_{j, \varepsilon}=\tau_{\varepsilon \boldsymbol{\xi}_{j}} \circ \nu^{j}=\tau_{\varepsilon \boldsymbol{\xi}_{j}} \circ\left(\mathcal{L}\left(\psi^{j} \vartheta\right)\right)=\mathcal{L} \vartheta_{j, \varepsilon},
$$

where $\varepsilon \in(0,1)$. Clearly, $\operatorname{supp} \nu_{j, \varepsilon} \subset \operatorname{supp} \vartheta_{j, \varepsilon} \subset \Omega$. We next define

$$
\nu_{\varepsilon}=\sum_{j=0}^{J} \nu_{j, \varepsilon}=\mathcal{L}\left(\sum_{j=0}^{J} \vartheta_{j, \varepsilon}\right) .
$$

We shall finally show that $\nu_{\varepsilon}$ converges to $\nu$ in the weak ${ }^{\star}$ sense of $\left(C^{\ell}(\bar{\Omega})\right)^{\prime}$. The sequence $\left\{\vartheta^{k}\right\}_{k \in \mathbb{N}}$ we seek can thus be defined by $\vartheta^{k}=\sum_{j=0}^{J} \vartheta_{j, k^{-1}}$.

We first show that the distribution $\nu^{j}=\mathcal{L}\left(\psi^{j} \vartheta\right)=\mathcal{L}\left(\psi^{j}(e * \nu)\right)$ defines an element of the dual of $C^{\ell}(\bar{\Omega})$, for every $j=0, \ldots, J$. We have that

$$
\nu^{j}=\mathcal{L}\left(\psi^{j} \vartheta\right)=\psi^{j} \mathcal{L} \vartheta+\sum_{|\beta|<m} \mathcal{L}_{\beta} \psi^{j} D^{\beta} \vartheta=\psi^{j} \nu+\sum_{|\beta|<m} \mathcal{L}_{\beta} \psi^{j}\left(D^{\beta} e\right) * \nu,
$$

where $\mathcal{L}_{\beta}$ is a linear partial differential operator with constant coefficients of order not exceeding $m-|\beta|$; thus $\mathcal{L}_{\beta} \psi^{j} \in \mathscr{D}\left(\mathbb{R}^{n}\right)$. It is clear that if $\mu \in\left(C^{\ell}(\bar{\Omega})\right)^{\prime}$ and $\varphi \in \mathscr{D}\left(\mathbb{R}^{n}\right)$, then $\varphi \mu \in\left(C^{\ell}(\bar{\Omega})\right)^{\prime}$, since $\varphi \mu(u)=\mu(\varphi u)$. Thus it suffices to show that the distributions $D^{\beta} e * \nu,|\beta|<m$, define continuous linear functionals on $C^{\ell}(\bar{\Omega})$. 
We know that each of the distributions $D^{\beta} e * \nu$ is supported in $\bar{\Omega}$. Next we need the following lemma:

Lemma 2. Let $\nu \in\left(C^{\ell}(\bar{\Omega})\right)^{\prime}$, where $\Omega$ is a bounded domain and $f \in L_{\mathrm{loc}}^{1}\left(\mathbb{R}^{n}\right)$. If the distribution $\mu=f * \nu$ is supported in $\bar{\Omega}$, then $\mu$ defines a continuous linear functional on $C^{\ell}(\bar{\Omega})$.

We postpone the proof of Lemma 2 for a while.

Proposition 1 and Lemma 2 provide that the distributions

$$
\nu^{j}=\mathcal{L}\left(\psi^{j} \vartheta\right)=\psi^{j} \nu+\sum_{|\beta|<m} \mathcal{L}_{\beta} \psi^{j} D^{\beta} \vartheta
$$

where $j=0, \ldots, J$, define elements of the dual of $C^{\ell}(\bar{\Omega})$.

Next, we shall show that

$$
\nu_{\varepsilon}=\sum_{j=0}^{J} \nu_{j, \varepsilon}=\sum_{j=0}^{J} \tau_{\varepsilon \boldsymbol{\xi}_{j}} \circ \nu^{j},
$$

converges to $\nu=\sum_{j=0}^{J} \nu^{j}$, in the weak ${ }^{\star}$ sense of $C^{\ell}(\bar{\Omega})$. It suffices to show that $\nu_{j, \varepsilon}$ converges to $\nu^{j}$, in the weak ${ }^{\star}$ sense of $C^{\ell}(\bar{\Omega})$, for every $j=0, \ldots, J$. We shall initially show that $\nu_{j, \varepsilon}=\tau_{\varepsilon \boldsymbol{\xi}_{j}} \circ \nu^{j}$ defines an element of $\left(C^{\ell}(\bar{\Omega})\right)^{\prime}$. It is readily seen that $\operatorname{supp}\left(\tau_{\varepsilon \boldsymbol{\xi}_{j}} \circ \nu^{j}\right) \subset \bar{\Omega}_{j}$, for every $\varepsilon \in(0,1)$, where $\Omega_{j}=\Omega \cap\left(\bigcup_{\varepsilon \in[0,1)} \tau_{\varepsilon \boldsymbol{\xi}_{j}}\left[U_{j}\right]\right)$, and if $\varphi \in C_{0}^{\ell}\left(\mathbb{R}^{n}\right)$, then

$$
\left|\tau_{\varepsilon \boldsymbol{\xi}_{j}} \circ \nu^{j}(\varphi)\right|=\left|\nu^{j}\left(\tau_{-\varepsilon \boldsymbol{\xi}_{j}} \circ \varphi\right)\right| \leq\left\|\nu^{j}\right\| \cdot\left|\tau_{-\varepsilon \boldsymbol{\xi}_{j}} \circ \varphi\right|_{\ell, \mathbb{R}^{n}}=\left\|\nu^{j}\right\| \cdot|\varphi|_{\ell, \mathbb{R}^{n}} .
$$

Here $\|\cdot\|$ is the norm in $\left(C_{0}^{\ell}\left(\mathbb{R}^{n}\right)\right)^{\prime}$, the dual of $C_{0}^{\ell}\left(\mathbb{R}^{n}\right)$. Thus $\tau_{\varepsilon \xi_{j}} \circ \nu^{j} \in\left(C_{0}^{\ell}\left(\mathbb{R}^{n}\right)\right)^{\prime}$, and since $\operatorname{supp}\left(\tau_{\varepsilon \boldsymbol{\xi}_{j}} \circ \boldsymbol{\nu}^{j}\right) \subset \bar{\Omega}_{j} \subset \bar{\Omega}$, then $\tau_{\varepsilon \boldsymbol{\xi}_{j}} \circ \nu^{j}$ defines an element of $\left(C^{\ell}\left(\bar{\Omega}_{j}\right)\right)^{\prime}$ as well as of $\left(C^{\ell}(\bar{\Omega})\right)^{\prime}$. If $u \in C^{\ell}(\bar{\Omega})$, then $\left.\tau_{-\varepsilon \xi_{j}} \circ u\right|_{\bar{\Omega}_{j}} \in C^{\ell}\left(\bar{\Omega}_{j}\right)$, for every $\varepsilon \in[0,1)$. The functionals $\lambda(u)=\tau_{\varepsilon \xi_{j}} \circ \nu^{j}(u)$ and $\mu(u)=\nu^{j}\left(\tau_{-\varepsilon \boldsymbol{\xi}_{j}} \circ u\right)$ are continuous on $C_{0}^{\ell}\left(\mathbb{R}^{n}\right)$ and they agree for every $u \in C_{0}^{\ell}\left(\mathbb{R}^{n}\right)$. Therefore, $\tau_{\varepsilon \boldsymbol{\xi}_{j}} \circ \nu^{j}(u)=\nu^{j}\left(\tau_{-\varepsilon \boldsymbol{\xi}_{j}} \circ u\right)$, for every $u \in C^{\ell}\left(\bar{\Omega}_{j}\right)$, and thus

$$
\left|\tau_{\varepsilon \boldsymbol{\xi}_{j}} \circ \nu^{j}(u)-\nu^{j}(u)\right|=\left|\nu^{j}\left(\tau_{-\varepsilon \boldsymbol{\xi}_{j}} \circ u\right)-\nu^{j}(u)\right| \leq\left\|\nu^{j}\right\| \cdot\left|\tau_{-\varepsilon \boldsymbol{\xi}_{j}} \circ u-u\right|_{\ell, \bar{\Omega}_{j}} .
$$

Here $\|\cdot\|$ is the norm of $\left(C^{\ell}\left(\bar{\Omega}_{j}\right)\right)^{\prime}$. Clearly, $\left|\tau_{-\varepsilon \xi_{j}} \circ u-u\right|_{\ell, \bar{\Omega}_{j}}$, tends to zero as $\varepsilon \searrow 0$, due to the uniform continuity of $D^{\alpha} u,|\alpha| \leq k$, in $\bar{\Omega}_{j}$.

Proof of Lemma 2, Since $\nu$ defines a distribution of compact support (i.e., $\operatorname{supp} \nu$ $\subset \bar{\Omega})$, then the convolution $\mu=f * \nu$ defines a distribution in $\mathbb{R}^{n}$. In order to prove that $\mu$ defines a continuous linear functional on $C^{\ell}(\bar{\Omega})$ it suffices to prove that $\mu$ defines a continuous linear functional on $C_{0}^{\ell}\left(\mathbb{R}^{n}\right)$, since it is given that $\operatorname{supp} \mu \subset \bar{\Omega}$. Note that $\mathscr{D}\left(\mathbb{R}^{n}\right)$ is dense in $C_{0}^{\ell}\left(\mathbb{R}^{n}\right)$, and thus it suffices to prove that there exists a $C>0$, such that

$$
|\langle\varphi, f * \nu\rangle| \leq C|\varphi|_{\ell, \mathbb{R}^{n}}, \quad \text { for every } \varphi \in \mathscr{D}\left(\mathbb{R}^{n}\right) .
$$

Let $\zeta \in \mathscr{D}\left(\mathbb{R}^{n}\right)$, which is equal to 1 in a neighborhood $\bar{\Omega}$. Then for every $\varphi \in \mathscr{D}\left(\mathbb{R}^{n}\right)$, we have that $\langle\zeta \varphi, f * \nu\rangle=\langle\varphi, f * \nu\rangle$, since $\operatorname{supp} f * \nu \subset \bar{\Omega}$. A standard property of the convolutions of distributions yields that ([42, Chapter 6$])$,

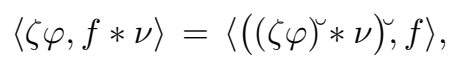


where $\breve{w}(\boldsymbol{x})=w(-\boldsymbol{x})$. Also, $(\zeta \varphi)^{u} * \nu \in \mathscr{D}$, and

$$
\left.\left((\zeta \varphi)^{\zeta}\right) * \nu\right)^{\zeta}(\boldsymbol{x})=\left((\zeta \varphi)^{\sqcup} * \nu\right)(-\boldsymbol{x})=\left(\tau_{\boldsymbol{x}} \nu\right)(\zeta \varphi)=\nu\left(\tau_{-\boldsymbol{x}}(\zeta \varphi)\right) .
$$

Next we have,

$$
\langle\zeta \varphi, f * \nu\rangle=\left\langle\nu\left(\tau_{-\boldsymbol{x}}(\zeta \varphi)\right), f\right\rangle=\int_{\mathbb{R}^{n}} \nu\left(\tau_{-\boldsymbol{x}}(\zeta \varphi)\right) f(\boldsymbol{x}) d \boldsymbol{x} .
$$

The last integral is well defined since $w(\boldsymbol{x})=\nu\left(\tau_{-\boldsymbol{x}}(\zeta \varphi)\right)$ is supported in $6=$ $\operatorname{supp} \zeta-\operatorname{supp} \nu$, which is compact. Thus,

$$
|\langle\varphi, f * \nu\rangle|=\left|\int_{K} \tau_{-\boldsymbol{x}}(\zeta \varphi) f(\boldsymbol{x}) d \boldsymbol{x}\right| \leq\left(\int_{K}|f(\boldsymbol{x})| d \boldsymbol{x}\right) \cdot \sup _{\boldsymbol{x} \in K}\left|\nu\left(\tau_{-\boldsymbol{x}}(\zeta \varphi)\right)\right| .
$$

Also,

$$
\sup _{\boldsymbol{x} \in K}\left|\nu\left(\tau_{-\boldsymbol{x}}(\zeta \varphi)\right)\right| \leq\|\nu\| \cdot|\zeta \varphi|_{\ell, \mathbb{R}^{n}} \leq c \cdot\|\nu\| \cdot|\varphi|_{\ell, \mathbb{R}^{n}}
$$

for a suitable $c>0$, where $\|\nu\|$ is the norm of $\nu$ in $\left(C_{0}^{\ell}\left(\mathbb{R}^{n}\right)\right)^{\prime}$. Altogether, for every $\varphi \in \mathscr{D}$, we have $|\langle\varphi, f * \nu\rangle| \leq C|\varphi|_{\ell, \mathbb{R}^{n}}$, where $C=c \cdot\|\nu\| \cdot \int_{K}|f(\boldsymbol{x})| d \boldsymbol{x}$.

\section{REFERENCES}

1. M. Abramowitz and I. A. Stegun (eds.), Handbook of mathematical functions with formulas, graphs, and mathematical tables, Dover Publications Inc., New York, 1992, Reprint of the 1972 edition. MR1225604 (94b:00012)

2. R. A. Adams and J. J. F. Fournier, Sobolev Spaces, 2nd ed., Academic Press, Amsterdam, 2003. MR 2424078

3. М. A. Aleksidze, K вопросу о практическом применении одного нового приближенного метода. (Russian) [On the question of a practical application of a new approximation method], Differencial'nye Uravnenija 2 (1966), 1625-1629. MR0203975 (34:3822)

4. — Фундаментальные функции в приближенных решениях граничных задач. (Russian) [Fundamental functions in approximate solutions of boundary value problems], "Nauka", Moscow, 1991, With an English summary. MR.1154556 (93c:65155)

5. E. Almansi, Sull'integrazione dell'equazione differenziale $\Delta^{2}=0$, Atti. Reale. Accad. Sci. Torino 31 (1896), 881-888.

6. Sull'integrazione dell'equazione differenziale $\Delta^{2 n}=0$, Annali di Mathematica Pura et Applicata, Series III 2 (1898), 1-51.

7. C. J. S. Alves, Inverse scattering with spherical incident waves, Mathematical and numerical aspects of wave propagation (Golden, CO, 1998), SIAM, Philadelphia, PA, 1998, pp. 502-504.

8. N. Aronszajn, T. M. Creese, and L. J. Lipkin, Polyharmonic functions, Oxford Mathematical Monographs, The Clarendon Press Oxford University Press, New York, 1983, Oxford Science Publications. MR745128 (86g:31001)

9. A. Bogomolny, Fundamental solutions method for elliptic boundary value problems, SIAM J. Numer. Anal. 22 (1985), no. 4, 644-669. MR795946 (87d:65143)

10. F. E. Browder, Approximation by solutions of partial differential equations, Amer. J. Math. 84 (1962), 134-160. MR0178247(31:2505)

11. C. S. Chen, M. Ganesh, M. A. Golberg, and A. H.-D. Cheng, Multilevel compact radial functions based computational schemes for some elliptic problems, Comput. Math. Appl. 43 (2002), no. 3-5, 359-378, Radial basis functions and partial differential equations. MR 1883573

12. A. H.-D. Cheng, H. Antes, and N. Ortner, Fundamental solutions of products of Helmholtz and polyharmonic operators, Eng. Anal. Bound. Elem. 14 (1994), no. 2, 187-191.

13. H. A. Cho, M. A. Golberg, A. S. Muleshkov, and X. Li, Trefftz methods for time dependent partial differential equations, Comput. Mat. Cont. 1 (2004), no. 1, 1-37.

14. A. Doicu, Y. Eremin, and T. Wriedt, Acoustic and Electromagnetic Scattering Analysis using Discrete Sources, Academic Press, New York, 2000.

15. L. Ehrenpreis, On the theory of kernels of Schwartz, Proc. Amer. Math. Soc. 7 (1956), 713718. MR0082637(18:584d)

\footnotetext{
${ }^{6}$ Here $A-B=\{x-y: x \in A$ and $y \in B\}$.
} 
16. G. Fairweather and A. Karageorghis, The method of fundamental solutions for elliptic boundary value problems. Numerical treatment of boundary integral equations, Adv. Comput. Math. 9 (1998), no. 1-2, 69-95. MR1662760(2000a:65165)

17. G. Fairweather, A. Karageorghis, and P. A. Martin, The method of fundamental solutions for scattering and radiation problems, Eng. Anal. Bound. Elem. 27 (2003), 759-769.

18. G. Fairweather, A. Karageorghis, and Y.-S. Smyrlis, A matrix decomposition MFS algorithm for axisymmetric biharmonic problems, Adv. Comput. Math. 23 (2005), no. 1-2, 55-71. MR 2131993 (2005m:65306)

19. G. B. Folland, Real analysis, 2nd ed., John Wiley \& Sons Inc., New York, 1999. MR1681462 (2000c:00001)

20. D. Gilbarg and N. S. Trudinger, Elliptic partial differential equations of second order, second ed., Grundlehren der Mathematischen Wissenschaften, vol. 224, Springer-Verlag, Berlin, 1983. MR.737190 (86c:35035)

21. M. A. Golberg and C. S. Chen, Discrete projection methods for integral equations, Computational Mechanics Publications, Southampton, 1997. MR1445293(98c:45002)

22. - The method of fundamental solutions for potential, Helmholtz and diffusion problems, Boundary integral methods: numerical and mathematical aspects, Comput. Eng., vol. 1, WIT Press/Comput. Mech. Publ., Boston, MA, 1999, pp. 103-176. MR1690853 (2000f:65107)

23. I. S. Gradshteyn and I. M. Ryzhik, Table of integrals, series, and products, 6 th ed., Academic Press, San Diego, 2000. MR.1773820 (2001c:00002)

24. L. Hörmander, The analysis of linear partial differential operators. I, Grundlehren der Mathematischen Wissenschaften, vol. 256, Springer-Verlag, Berlin, 1983, Distribution theory and Fourier analysis. MR717035 (85g:35002a)

25. A. Karageorghis and G. Fairweather, The method of fundamental solutions for the numerical solution of the biharmonic equation, J. Comput. Phys. 69 (1987), no. 2, 434-459. MR888063 (88f:65219)

26. The Almansi method of fundamental-solutions for solving biharmonic problems, Int. J. Numer. Meth. Engng. 26 (1988), no. 7, 1665-1682.

27. M. Katsurada, A mathematical study of the charge simulation method. II, J. Fac. Sci. Univ. Tokyo Sect. IA Math. 36 (1989), no. 1, 135-162. MR.991024(90d:35061)

28. _ Asymptotic error analysis of the charge simulation method in a Jordan region with an analytic boundary, J. Fac. Sci. Univ. Tokyo Sect. IA Math. 37 (1990), no. 3, 635-657. MR 1080874 (91m:65263)

29. T. Kitagawa, On the numerical stability of the method of fundamental solution applied to the Dirichlet problem, Japan J. Appl. Math. 5 (1988), no. 1, 123-133. MR.924746 (89a:65152)

30. J. A. Kołodziej, Zastosowanie metody kollokacji brzegowej w zagadnieniach mechaniki. (Polish) [Applications of the Boundary Collocation Method in Applied Mechanics], Wydawnictwo Politechniki Poznańskiej, Poznań, 2001.

31. V. D. Kupradze, On a method of solving approximately the limiting problems of mathematical physics, Ž. Vyčisl. Mat. i Mat. Fiz. 4 (1964), 1118-1121. MR0174183 (30:4390)

32. _ Potential methods in the theory of elasticity, Translated from the Russian by H. Gutfreund. Translation edited by I. Meroz, Israel Program for Scientific Translations, Jerusalem, 1965. MR0223128 (36:6177)

33. V. D. Kupradze and M. A. Aleksidze, An approximate method of solving certain boundaryvalue problems, Soobšč. Akad. Nauk Gruzin. SSR 30 (1963), 529-536, in Russian. MR0155098 $(27: 5039)$

34. V. D. Kupradze, T. G. Gegelia, M. O. Basheleshvili, and T. V. Burchuladze, Трехмерные задачи математической теории упругости и термоупругости. (Russian) [Three-dimensional problems in the mathematical theory of elasticity and thermoelasticity.], Izdat. "Nauka", Moscow, 1976.

35. P. K. Kythe, Fundamental solutions for differential operators and applications, Birkhäuser, Boston, MA, 1996. MR.1395370 (97f:00009)

36. A. E. H. Love, A Treatise on the Mathematical Theory of Elasticity, Dover Publications, New York, 1944, 4th Ed. MR0010851 (6:79e)

37. M. Maiti and S. K. Chakrabarty, Integral equation solutions for simply supported polygonal plates, Int. J. Engng. Sci. 12 (1974), no. 10, 793-806. 
38. B. Malgrange, Existence et approximation des solutions des équations aux dérivées partielles et des équations de convolution, Ann. Inst. Fourier, Grenoble 6 (1955-1956), 271-355. MR0086990 (19:280a)

39. R. Mathon and R. L. Johnston, The approximate solution of elliptic boundary-value problems by fundamental solutions, SIAM J. Numer. Anal. 14 (1977), no. 4, 638-650. MR0520337 $(58: 25006)$

40. S. N. Mergelyan, Равномерные приближения функций комплексного переменного. (Russian) [Uniform approximations of functions of a complex variable], Uspehi Matem. Nauk (N.S.) 7 (1952), no. 2(48), 31-122. MR0051921 (14:547e)

41. M. Nicolescu, Les fonctions polyharmoniques, Hermann, Paris, 1936.

42. W. Rudin, Functional Analysis, McGraw-Hill, New York, 1973. MR0365062 (51:1315)

43. C. Runge, Zur Theorie der eindeutigen analytischen Funktionen, Acta Math. 6 (1885), 229244. MR 1554664

44. L. Schwartz, Théorie des distributions. 2 Tomes, Actualités Sci. Ind., no. 1091, $1122=$ Publ. Inst. Math. Univ. Strasbourg 9-10, Hermann \& Cie., Paris, 1950-1951. MR0035918 (12:31d)

45. - The method of fundamental solutions: a weighted least-squares approach, BIT 46 (2006), no. 1, 163-194. MR2214854 (2006m:65292)

46. Y.-S. Smyrlis, Mathematical foundation of the MFS for certain elliptic systems in linear elasticity, Numer. Math. (2009), DOI: 10.1007/s00211-008-0207.1.

47. A A A proximations by solutions of elliptic equations in semilocal spaces, J. Math. Anal. Appl. 350 (2009), no. 1, 122-134.

48. Y.-S. Smyrlis and A. Karageorghis, The under-determined version of the MFS: Taking more sources than collocation points, Submitted for publication.

49. — A linear least-squares MFS for certain elliptic problems, Numer. Algorithms 35 (2004), no. 1, 29-44. MR2041801 (2004m:65173)

50. - Numerical analysis of the MFS for certain harmonic problems, M2AN Math. Model. Numer. Anal. 38 (2004), no. 3, 495-517. MR2075757 (2005k:65224)

51. P. Sundqvist, Numerical Computations with Fundamental Solutions (Numeriska beräkingar med fundamentallösningar), Ph.D. thesis, University of Uppsala, Faculty of Science and Technology, May 2005.

52. N. N. Tarkhanov, The Cauchy problem for solutions of elliptic equations, Akademie Verlag, Berlin, 1995. MR1334094 (96d:35024)

53. E. Trefftz, Ein Gegenstück zum Ritzschen Verfahren, $2^{\text {er }}$ Intern. Kongr. für Techn. Mech., Zürich, 1926, pp. 131-137.

54. F. Trèves, Linear partial differential equations with constant coefficients: Existence, approximation and regularity of solutions, Mathematics and its Applications, Vol. 6, Gordon and Breach Science Publishers, New York, 1966. MR0224958 (37:557)

55. Th. Tsangaris, Y.-S. Smyrlis, and A. Karageorghis, Numerical analysis of the method of fundamental solutions for harmonic problems in annular domains, Numer. Methods Partial Differential Equations 22 (2006), no. 3, 507-539. MR.2212224 (2006m:65289)

56. T. Ushijima and F. Chiba, Error estimates for a fundamental solution method applied to reduced wave problems in a domain exterior to a disc, J. Comput. Appl. Math. 159 (2003), no. 1, 137-148. MR2022324

57. B. M. Weinstock, Uniform approximation by solutions of elliptic equations, Proc. Amer. Math. Soc. 41 (1973), 513-517. MR0340794 (49:5544)

Department of Mathematics and Statistics, University of Cyprus, (Пaneinsthmio

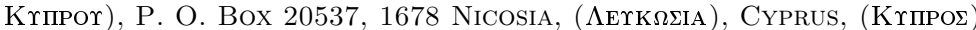

E-mail address: smyrlis@ucy.ac.cy 